\title{
A Farewell to Liouvillians
}

\author{
Vadim Oganesyan ${ }^{1}$, J. T. Chalker ${ }^{2}$, and S. L. Sondhi ${ }^{1}$ \\ ${ }^{1}$ Deptartment of Physics, Princeton University, Princeton, NJ 08544, USA and \\ 2 Theoretical Physics, University of Oxford, 1 Keble Road, Oxford, OX1 3NP, United Kingdom
}

(Dated: October 31, 2018)

\begin{abstract}
We examine the Liouvillian approach to the quantum Hall plateau transition, as introduced recently by Sinova, Meden, and Girvin [Phys. Rev. B 62, 2008 (2000)] and developed by Moore, Sinova and Zee [Phys. Rev. Lett. 87, 046801 (2001)]. We show that, despite appearances to the contrary, the Liouvillian approach is not specific to the quantum mechanics of particles moving in a single Landau level: we formulate it for a general disordered single-particle Hamiltonian. We next examine the relationship between Liouvillian perturbation theory and conventional calculations of disorder-averaged products of Green functions and show that each term in Liouvillian perturbation theory corresponds to a specific contribution to the two-particle Green function. As a consequence, any Liouvillian approximation scheme may be re-expressed in the language of Green functions. We illustrate these ideas by applying Liouvillian methods, including their extension to $N_{L}>1$ Liouvillian flavors, to random matrix ensembles, using numerical calculations for small integer $N_{L}$ and an analytic analysis for large $N_{L}$. We find that behavior at $N_{L}>1$ is different in qualitative ways from that at $N_{L}=1$. In particular, the $N_{L}=\infty$ limit expressed using Green functions generates a pathological approximation, in which two-particle correlation functions fail to factorize correctly at large separations of their energy, and exhibit spurious singularities inside the band of random matrix energy levels. We also consider the large $N_{L}$ treatment of the quantum Hall plateau transition, showing that the same undesirable features are present there, too.
\end{abstract}

\section{INTRODUCTION}

It has long been appreciated that the quantum Hall effect depends for its existence on localization by disorder of quasiparticles in Landau level tails, and that neighboring quantum Hall plateaus are separated by a continuous quantum phase transition characterized by a diverging localization length.1 The scaling ideas which encapsulate this understanding 23 are supported by extensive numerical strdiest and by some of the available experimental datat 5 althqugh the full experimental situation remains unsettled 6 . An analytic theory of the transition, however, has proved elusive, even for the simplest models which include disorder and magnetic field but omit electron-electron interactions.

In this context, two successive recent developments have attracted interest: Sinova, Meden and Girvind (SMG) have introduced a Liouvillian approach to localization if the lowest Landau level, which Moore, Sinova and Zee (MSZ) have extended, with the introduction of $N_{L}$ Liouvillian flavors and an expansion in powers of $1 / N_{L}$ about the large $N_{L}$ limit 9 . In brief, the Liouvillian is the time evolution operator for electron probability density, and the information it encodes on localization is integrated over states at all energies within the disorder-broadened Landau level. Despite the energy integration, the critical behavior of the localization length at the plateau transition can, in principle, be extracted from the dependence of the Liouvillian propagator on frequency $\omega$, provided this is known sufficiently accurately (see SMG and also Appendix A of this paper). The original work of SMG reported a calculation of the Liouvillian propagator using a version of the self-consistent Born approximation (SCBA), which is exact for the $N_{L}=\infty$ limit of MSZ, and yields diffusive time evolution without localization. MSZ have conjectured that the $\mathcal{O}\left(1 / N_{L}\right)$ correction, which is logarithmically divergent at small $\omega$, may be used to arrive at an estimate for the localization length exponent $\nu$.

Prima facie there are three reasons to think that this work may have outflanked the obstructions faced by previous approaches. First, the derivation of the Liouvillian formalism by SMG invokes the algebra of density operators projected onto the lowest Landau level, thus appearing to build in the physics of high magnetic fields at the first step. Second, the formalism deals directly with a disorder-averaged two-particle quantity, avoiding intermediate calculations of a one-particle Green function, and hence, plausibly, goes directly to the heart of the matter. Third, the extension to $N_{L}$ Liouvillian flavors is distinct from the standard extension to $N$ orbitals in localization problems, 10 which is known to capture weak localization physics but not the quantum Hall delocalization transition 13. This gives grounds for hope that the logarithm found by MSZ may not simply be due to weak localization effects and might indeed be used to get an estimate for the correlation length exponent.

Given the potential importance of these developments, it seems useful to investigate the Liouvillian approach in some detail and to relate it to established methods. This is our objective in the present paper. There are four distinct facets to our results. First, we show that although the Liouvillian approach has appeared in past work to be tailored specifically to the quantum mechanics of particles moving in a single Landau level, since it makes use of the algebra of projected density operators, it can in fact be formulated for any disordered single-particle Hamiltonian. Second, we compare the perturbation expansion for the Liouvillian propagator with conventional calcu- 
lations of the disorder-averaged two-particle Green function. We demonstrate that each term in the perturbation expansion for the Liouvillian propagator corresponds to a specific combination of terms in the Green function expansion. As a consequence, any approximation scheme within one approach has an equivalent in the other approach. Moverover, it is possible to translate between the two approaches in either direction: to go from the twoparticle Green function to the Liouvillian simply involves an energy integration, while a more elaborate proceedure, which we set out, is required to undo this energy integration and pass in the opposite direction. Third, we illustrate these ideas by applying the Liouvillian approach to random matrix theory, discussing the Gaussian unitary and orthogonal ensembles. We obtain an analytic solution in the limit of large flavor number $N_{L}$, for arbitrary matrix size $N$, and we supplement this with numerical calculations for finite $N_{L}$, finding qualitative differences between results at $N_{L}=\infty$ (and all $N_{L}>1$ ) and those at $N_{L}=1$. We also undo the energy average at $N_{L}=\infty$, showing that the Liouvillian SCBA has a very different character from established approximations when translated into a calculation for the two-particle Green function. It constitutes an approximation without the usual structure based on single-particle self-energies and twoparticle irreducible vertices. Disappointingly, this is not progress as the approximation is pathological: the resulting Green functions exhibit spurious singularities inside the band and fail to factorize correctly at large energy separations. Finally, we return to the plateau transition. Progress in this case is more difficult, because even the Liouvillian SCBA of SMG requires a numerical solution. We are nevertheless able to show that the two-particle Green function generated by the Liouvillian SCBA has undesirable features in this case, too, failing to factorise as it should for large separations between pairs of its spatial arguments, and exhibiting spurious singularities as a function of energy in this limit.

In sum, on one hand we have shown generally that any Liouvillian approximation can equally well be expressed using the better-understood machinery of Green functions, and on the other hand we have argued that the only existing basis for Liouvillian calculations, the $1 / N_{L}$ expansion, is seriously flawed. In combination, these results leave us pessimistic about the scope for advances in the theory of the quantum Hall plateau transition using Liouvillian methods.

In the balance of the paper we set out the technical content of these assertions. In Section II we develop Liouvillian machinery in a general setting and describe, first, how to go from two particle Green functions to Liouvillians by energy integration, and second, our algorithm for undoing this energy integration within a given Liouvillian approximation. In Section III we review the $1 / N_{L}$ expansion scheme introduced by MSZ, again in a general setting. Section IV is devoted to a detailed examination of the Liouvillian technique applied to the test case of random matrix statistics. In Section $\mathrm{V}$ we consider the quantum Hall problem and describe the pathology that is immediately apparent by recourse to the previous results. We end with a summary and three appendices which provide: a more careful discussion of the critical behavior of the quantum Hall Liouvillian than is available in previous work; some details omitted in the main text; and a construction that yields an algebra for an arbitrary single-particle Hamiltonian that is identical to the algebra of density operators projected onto the lowest Landau level.

\section{GENERAL CONSIDERATIONS}

\section{A. From two-particle Green functions to the Liouvillian}

Consider a single-particle Hamiltonian $\hat{H}$ acting on basis states $|a\rangle$ in a space $\mathcal{V}$ of dimensionality $N$. Although all of its properties are encapsulated in the corresponding one-particle Green functions

$$
G_{a b}^{ \pm}(E)=\left\langle a\left|\frac{1}{E-\hat{H} \pm i \delta}\right| b\right\rangle,
$$

(where $\delta$ is a positive infinitesimal), in most problems of interest their more easily computed disorder averages $\left\langle G^{ \pm}\right\rangle$have little of the interesting information contained in $G^{ \pm}$. One is then forced to consider higher order correlators such as the two-particle retarded-advanced Green function

$$
K_{a A ; b B}^{+-}(E, \omega)=G_{a b}^{+}\left(E+\frac{\omega}{2}\right) G_{B A}^{-}\left(E-\frac{\omega}{2}\right),
$$

whose disorder average does contain useful information. Analogously one can define $K^{++}, K^{-+}$and $K^{--}$. Here and in the following, we use \pm as superscripts to indicate retarded and advanced Green functions, and reserve lower case state labels $(a, b)$ for the former, and upper case ones $(A, B)$ for the latter.

The central object in this paper is the energy integral of $K_{a A ; B B}^{+-}(E, \omega)$

$$
\Pi_{a A ; b B}(\omega) \equiv \int_{-\infty}^{\infty} \frac{d E}{2 \pi i} K_{a A ; b B}^{+-}(E, \omega) .
$$

We will now show that, quite generally, this can be expressed as the one particle Green function of a Liouville "super-operator" (the Liouvillian) which itself acts on the space $\mathcal{A}_{\mathcal{V}}$ of all linear operators on the space $\mathcal{V}$. Clearly, this Liouvillian Green function is to be distinguished from the usual Green function of the Hamiltonian, introduced in Eq. (11). The space $\mathcal{A}_{\mathcal{V}}$ is $N^{2}$ dimensional and is spanned by the basis set $|a\rangle\langle b|$. To emphasize that these operators themselves form a linear space, we will use the notation $\mid a, b)=|a\rangle\langle b|$. A natural inner product on this space is defined as $\operatorname{Tr} P^{\dagger} Q$ for any operators $P$ and $Q$ that belong to $\mathcal{A}_{\mathcal{V}}$. In terms of the basis this yields

$$
(a, b \mid c, d) \equiv \operatorname{Tr}\left\{[|a\rangle\langle b|]^{\dagger}|c\rangle\langle d|\right\}=\langle a \mid c\rangle\langle d \mid b\rangle .
$$


Finally, pairs of operators $P, Q$ define a super-operator on $\mathcal{A}_{\mathcal{V}}$ via the action $\left.P \circ Q \mid a, b\right)=P|a\rangle\langle b| Q$ from left and right respectively.
Now consider rewriting Eq. (3) (with tildes denoting inverse Fourier transforms)

$$
\begin{aligned}
\Pi_{a A ; b B}(\omega) & =\int_{-\infty}^{\infty} \frac{d E}{2 \pi i} G_{a b}^{+}\left(E+\frac{\omega}{2}\right) G_{B A}^{-}\left(E-\frac{\omega}{2}\right) \\
& =\int_{-\infty}^{\infty} \frac{d E}{2 \pi i} \int_{-\infty}^{\infty} d t_{1} \int_{-\infty}^{\infty} d t_{2} e^{i(E+\omega / 2+i \delta) t_{1}+i(E-\omega / 2-i \delta) t_{2}} \tilde{G}_{a b}^{+}\left(t_{1}\right) \tilde{G}_{B A}^{-}\left(t_{2}\right) \\
& =-i \int_{-\infty}^{\infty} d t e^{i(\omega+i \delta) t} \tilde{G}_{a b}^{+}(t) \tilde{G}_{B A}^{-}(-t)=-i \int_{-\infty}^{\infty} d t e^{i(\omega+i \delta) t}(\theta(t))^{2}\left\langle a\left|e^{-i H t}\right| b\right\rangle\left\langle B\left|e^{i H t}\right| A\right\rangle \\
& =-i \int_{-\infty}^{\infty} d t e^{i(\omega+i \delta) t} \theta(t) \operatorname{Tr}\left\{\left(e^{i H t}|A\rangle\langle a| e^{-i H t}\right)|b\rangle\langle B|\right\} \equiv-i \int_{0}^{\infty} d t e^{i(\omega+i \delta) t}(a, A \mid b, B)_{0}
\end{aligned}
$$

The single particle Hamiltonian induces a time evolution super-operator, the Liouvillian $\mathcal{L}$, on $\mathcal{A}_{\mathcal{V}}$

$$
\left.\mid a, A)_{t}=e^{i \mathcal{L} t} \mid a, A\right)_{0}
$$

and the matrix elements of $\mathcal{L}$ can be read off from

$$
\frac{d}{d t}|a\rangle\left\langle A\left|=i[\hat{H},|a\rangle\langle A|]=i \sum_{b B}\left(H_{a b} \delta_{B A}-\delta_{a b} H_{B A}\right)\right| b\right\rangle\left\langle B\left|\equiv i \sum_{b B} \mathcal{L}_{a A ; b B}\right| b\right\rangle\langle B|
$$

More abstractly,

$$
\hat{\mathcal{L}}=\hat{H} \circ \hat{1}-\hat{1} \circ \hat{H} .
$$

In terms of $\mathcal{L}$ we have finally

$$
\Pi_{a A ; b B}(\omega)=\left(a, A\left|\frac{1}{\omega-\hat{\mathcal{L}}+i \delta}\right| b, B\right)
$$

which is the promised rewriting of $\Pi_{a A ; b B}(\omega)$. While Eq. (8) does not appear in the work of SMG and MSZ, our $\mathcal{L}$ is simply the generalisation for arbitrary $\hat{H}$ of the Liouvillian discussed for the quantum Hall plateau transition in previous papers. We continue our general discussion below and take up the plateau transition in Section $\mathrm{V}$.

\section{B. Exact properties of $\hat{\mathcal{L}}$}

A few general and exact properties of $\hat{\mathcal{L}}$ can be inferred from the preceding formulae. The eigenvalues $\lambda_{a b}$ of $\hat{\mathcal{L}}$ are simply related to the eigenvalues $\epsilon_{a}$ of $\hat{H}$ by

$$
\lambda_{a b}=\epsilon_{a}-\epsilon_{b}
$$

This implies that $\lambda_{a b}=-\lambda_{b a}$, so that the eigenvalues of $\hat{\mathcal{L}}$ occur in pairs symmetric about $\lambda=0$. In addition, the
Liouvillian has at least $N$ zero eigenvalues, since $\lambda_{m m}=$ 0 . Finally, if the eigenvalues of $\hat{H}$ occupy a band of width $W$, the bandwidth of $\hat{\mathcal{L}}$ is $2 W$.

\section{Perturbation theory}

From Eq. (9) we can generate the perturbation expansion

$$
\Pi_{a A ; b B}(\omega)=\sum_{n=0}^{\infty} \frac{\left(a, A\left|\hat{\mathcal{L}}^{n}\right| b, B\right)}{(\omega+i \delta)^{n+1}}
$$

It is interesting to see how this arises from energy integrating the perturbative expression for the two-particle Green function. Consider the contribution to $K_{a A ; b B}^{+-}(\omega)$ at order $n$ in $\hat{H}$, which is

$K_{a A ; b B}^{(n)}(E, \omega)=\sum_{m=0}^{n} \frac{\left[\hat{H}^{m}\right]_{a b}\left[\hat{H}^{n-m}\right]_{B A}}{\left(E-\frac{\omega}{2}-i \delta\right)^{m+1}\left(E+\frac{\omega}{2}+i \delta\right)^{n+1-m}}$

Integration over $E$, 


$$
\int_{-\infty}^{\infty} K_{a A ; b B}^{(n)}(E, \omega) \frac{d E}{2 \pi i}=\frac{1}{(\omega+i \delta)^{n+1}} \sum_{m=0}^{n} \frac{(-1)^{m} n !}{(n-m) ! m !}\left[\hat{H}^{m}\right]_{a b}\left[\hat{H}^{n-m}\right]_{B A}
$$

produces precisely the corresponding term in the expansion for $\hat{\Pi}(\omega)$.

\section{Disorder averaging}

For the Green function perturbation theory with Gaussian randomness in $\hat{H}$, the effect of disorder averaging is to replace $\left\langle\hat{H}^{2 n}\right\rangle$ with a sum of products of all pairwise contractions $\langle\hat{H} \hat{H}\rangle$. We can translate this into the language of the Liouvillian theory by using the definition of $\hat{\mathcal{L}}$ to express its matrix elements in terms of those of $\hat{H}$, then using the correlators for the matrix elements of $\hat{H}$ to obtain those for $\hat{\mathcal{L}}$,

$$
\begin{aligned}
\left\langle\mathcal{L}_{a A ; b B} \mathcal{L}_{c C ; d D}\right\rangle & =\left\langle\left(\delta_{a b} H_{B A}-H_{a b} \delta_{B A}\right)\left(\delta_{c d} H_{D C}-H_{c d} \delta_{D C}\right)\right\rangle \\
& =\delta_{a b} \delta_{c d}\left\langle H_{B A} H_{D C}\right\rangle-\delta_{a b} \delta_{D C}\left\langle H_{B A} H_{c d}\right\rangle-\delta_{B A} \delta_{c d}\left\langle H_{a b} H_{D C}\right\rangle+\delta_{B A} \delta_{D C}\left\langle H_{a b} H_{c d}\right\rangle
\end{aligned}
$$

and finally using these to average over the powers $\hat{\mathcal{L}}^{2 n}$ that occur in the perturbative expansion.

\section{E. From Liouvillian perturbation theory to Green function perturbation theory}

The purpose of this subsection is to construct an algorithm for passing from a given (likely approximate) expression for $\hat{\Pi}(\omega)$ to one for $\hat{K}(E, \omega)$. Specifically, we would like to associate uniquely each disorder averaged diagram for $\hat{\Pi}(\omega)$ with a corresponding set involving Green functions. To make the reader's task simpler we we first summarize the basic idea of the algorithm, then provide a representative example, and finally state the general formula.

\section{Basic idea}

In going between Eq. (12) and Eq. (13), we have lost the distinction between advanced and retarded propogators (since $E \pm \frac{\omega}{2} \pm i \delta$ have been traded for $\omega$ ) but the matrix elements still record where the original disorder lines were attached in the two-particle Green function diagram. If we reconstruct this information from the Liouvillian matrix elements, where it must reside since they are defined by matrix elements of the Hamiltonian, our remaning task is merely one of undoing the signs and combinatorial factors introduced by the energy integration. This is easier done than said.11

\section{Example}

Let us see how this works at lowest non-trivial order (fourth, since at second order there is only one Liouvillian diagram). Of the three diagrams in Fig. 11 we will use

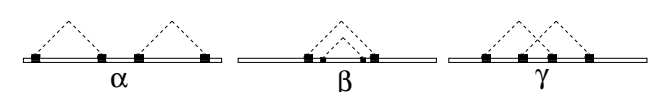

FIG. 1: Diagrams for $\hat{\Pi}(\omega)$ at fourth order in $\mathcal{L}$.

the middle one, denoting its contribution to $\Pi_{a A ; b B}(\omega)$ by $\Pi_{a A ; b B}^{(\beta)}(\omega)$. In terms of $\hat{H}$ it is (summation over all repeated indices is implied)

$$
\begin{aligned}
\Pi_{a A ; b B}^{(\beta)}(\omega)= & \frac{1}{(\omega+i \delta)^{5}}\left\langle\mathcal{L}_{a A ; u U} \mathcal{L}_{w W ; b B}\right\rangle\left\langle\mathcal{L}_{u U, v V} \mathcal{L}_{v V ; w W}\right\rangle \\
= & \frac{1}{(\omega+i \delta)^{5}}\left[\delta_{a b}\left\langle H_{B W} H_{U A}\right\rangle\left\langle\hat{H}^{2}\right\rangle_{W U}+\delta_{B A}\left\langle H_{a u} H_{w b}\right\rangle\left\langle\hat{H}^{2}\right\rangle_{u w}\right. \\
& -2\left\langle H_{a u} H_{v b}\right\rangle\left\langle H_{B A} H_{u v}\right\rangle-2\left\langle H_{a b} H_{V U}\right\rangle\left\langle H_{B V} H_{U A}\right\rangle \\
& -\left\langle H_{a u} H_{B A}\right\rangle\left\langle H^{2}\right\rangle_{u b}-\left\langle H_{a b} H_{B U}\right\rangle\left\langle H^{2}\right\rangle_{U A}-\left\langle H^{2}\right\rangle_{a u}\left\langle H_{u b} H_{B A}\right\rangle-\left\langle H_{a b} H_{U A}\right\rangle\left\langle H^{2}\right\rangle_{B U} \\
& \left.+2\left\langle\hat{H}^{2}\right\rangle_{a b}\left\langle\hat{H}^{2}\right\rangle_{B A}+4\left\langle H_{a u} H_{B U}\right\rangle\left\langle H_{u b} H_{U A}\right\rangle\right]
\end{aligned}
$$


which can be represented graphically by drawing the bare Liouvillian propagator as a double line and resolving disorder vertices using the Hamiltonian as indicated in Fig. 2. This is done in detail in Fig. 3, in which the first diagram is Eq. (15) and last ten are Eq. (16). The final

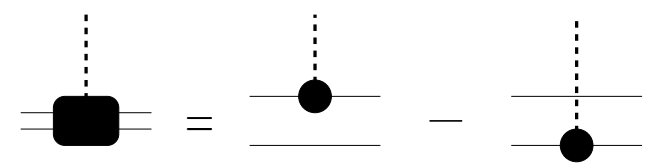

FIG. 2: Graphical representation of $\hat{\mathcal{L}}=\hat{H} \circ \hat{1}-\hat{1} \circ \hat{H}$.

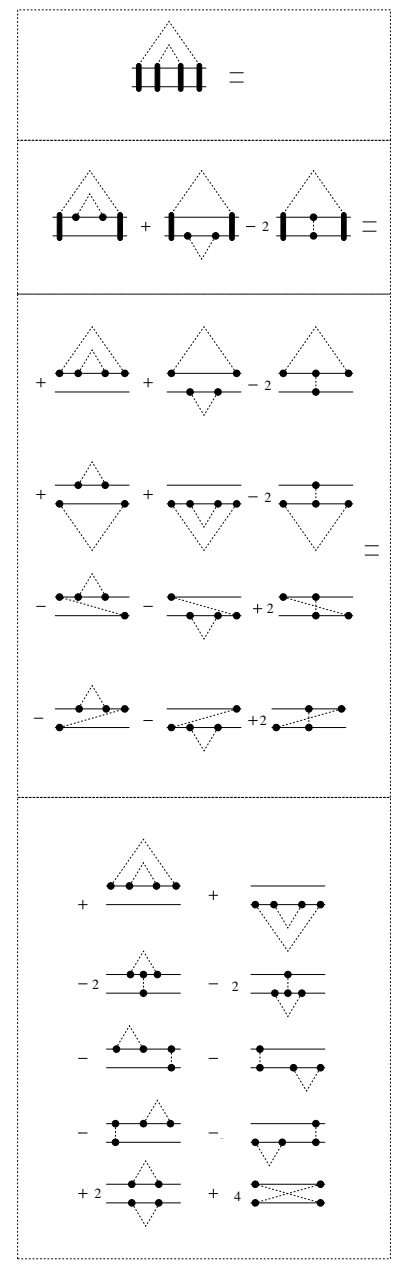

FIG. 3: Graphical representation of the correspondence between Liouvillian and Green function perturbation series. First diagram corresponds to Eq. 15 and contains two Liouvillian contractions. Next, three diagrams are obtained by redrawing one of the contractions using Fig. 2, each of which is in turn decomposed by repeating the procedure on the remaining Liouvillian contraction. Finally, the last ten diagrams represent Eq. 16.

step is to undo the signs and combinatorial factors generated by the energy integration in going from Eq. (12) to Eq. (13). For each term in our example, this may be done by simply counting how many times $H$ appears on upper

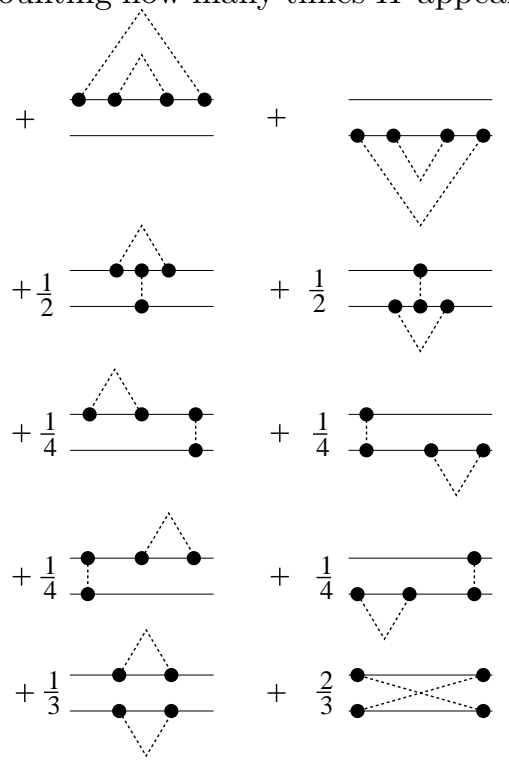

FIG. 4: Green functions for the Liouvillian diagram $\beta$ of Fig. 1. represented by Eqns. (16) and (17). This figure should be compared with the last ten diagrams of Fig. 3.

or lower lines, representing retarded or advanced Green functions. In this way, writing $E_{ \pm} \equiv E \pm \omega / 2 \pm i \delta$, we find that Eq. 15) generates a contribution to $K_{a A ; b B}^{ \pm}(E, \omega)$ of

$$
\begin{aligned}
K_{a A ; b B}^{(\beta)}(E, \omega)= & \frac{\delta_{a b}\left\langle H_{B W} H_{U A}\right\rangle\left\langle H^{2}\right\rangle_{W U}}{E_{+}^{5} E_{-}}+\frac{\delta_{B A}\left\langle H_{a u} H_{w z}\right\rangle\left\langle H^{2}\right\rangle_{u w}}{E_{+} E_{-}^{5}} \\
& -2 \times \frac{-1}{4} \times\left(\frac{\left\langle H_{a u} H_{v b}\right\rangle\left\langle H_{B A} H_{u v}\right\rangle}{E_{+}^{2} E_{-}^{4}}+\frac{\left\langle H_{a b} H_{V U}\right\rangle\left\langle H_{B V} H_{U A}\right\rangle}{E_{+}^{4} E_{-}^{2}}\right) \\
& -\frac{-1}{4} \times\left(\frac{\left\langle H_{a u} H_{B A}\right\rangle\left\langle H^{2}\right\rangle_{u b}}{E_{+}^{2} E_{-}^{4}}+\frac{\left\langle H_{a b} H_{B U}\right\rangle\left\langle H^{2}\right\rangle_{U A}}{E_{+}^{4} E_{-}^{2}}+\frac{\left\langle H^{2}\right\rangle_{a u}\left\langle H_{u b} H_{B A}\right\rangle}{E_{+}^{2} E_{-}^{4}}+\frac{\left\langle H_{a b} H_{U A}\right\rangle\left\langle H^{2}\right\rangle_{B U}}{E_{+}^{4} E_{-}^{2}}\right)
\end{aligned}
$$




$$
+2 \times \frac{1}{6} \times \frac{\left\langle H^{2}\right\rangle_{a b}\left\langle H^{2}\right\rangle_{B A}}{E_{+}^{3} E_{-}^{3}}+4 \times \frac{1}{6} \times \frac{\left\langle H_{a u} H_{B U}\right\rangle\left\langle H_{u b} H_{U A}\right\rangle}{E_{+}^{3} E_{-}^{3}}
$$

This equation is depicted graphically in Fig.4.

Three aspects of this exercise are worth noting. First, a given Liouvillian diagram contains a partial sum of several Green function diagrams. Second, the Green function diagrams are all added with positive weights. Third, one's conventional intuition about the importance of diagrams in Green function perturbation theory is suspect when carried over to Liouvillian perturbation theory. In this example, a seemingly simple, non-crossing Liouvillian diagram actually sums some of the crossing diagrams in Green function perturbation theory.

\section{The algorithm}

To apply the method illustrated above to all diagrams we need to automate the procedure for keeping track of the topology of disorder contractions. This can be accomplished by deforming the problem defined by Eqns. (8) and (9) to the form

$$
\begin{aligned}
\hat{\mathcal{L}}(p, h) & =p \hat{H} \circ \hat{1}-h \hat{1} \circ \hat{H} \\
\hat{\Pi}(\omega, p, h) & =[\omega-\hat{\mathcal{L}}(p, h)+i \delta]^{-1} .
\end{aligned}
$$

Here, $p$ and $h$ are arbitrary parameters which record whether $H$ acts on a retarded or an advanced Green function line. Consider any particular Liouvillian diagram at order $2 n$, which we label below with $\mu$. Its contribution to $\Pi(\omega)$ is

$$
\frac{\left\langle\hat{L}^{2 n}\right\rangle_{\mu}}{(\omega+i \delta)^{2 n+1}}=\sum_{m \alpha} A_{m \alpha}^{(\mu)} p^{2 n-m} h^{m} \frac{\left\langle\hat{H}^{m} \hat{H}^{2 n-m}\right\rangle_{\alpha \mu}}{(\omega+i \delta)^{2 n+1}},
$$

where $A_{m \alpha}^{(\mu)}$ are combinatorial coefficients and \langle\rangle$_{\mu}$ denotes a particular subset of Wick contractions of $\left\langle\hat{\mathcal{L}}^{2 n}\right\rangle$ corresponding to $\mu$ (and similarly for \langle\rangle$_{\alpha \mu}$ in the case of $\left.\left\langle\hat{H}^{2 n}\right\rangle\right)$. The diagram gives rise to a contribution to $K_{a A ; b B}^{ \pm}(E, \omega)$ of

$$
\sum_{m \alpha} A_{m \alpha}^{(\mu)}(-1)^{m} \frac{m !(2 n-m) !}{(2 n) !} \frac{\left\langle\hat{H}^{m} \hat{H}^{2 n-m}\right\rangle_{\alpha \mu}}{E_{+}^{m+1} E_{-}^{2 n-m+1}} .
$$

This step can be automated by making the substitutions: $\omega+i \delta \rightarrow E_{+} E_{-}, p \rightarrow x E_{+} / z$, and $h \rightarrow-y E_{-} / z$, and then, to attach the binomial coefficients, resorting to the identity

$$
\int_{0}^{\infty} d x \int_{0}^{\infty} d y \oint \frac{d z}{2 \pi i z} \exp (-x-y+z) \frac{x^{m} y^{n}}{z^{l}}=\frac{m ! n !}{l !}
$$

where the $z$ integration contour encloses the origin once in the anticlockwise direction. Thus the algorithm for energy un-integrating the Liouvillian perturbation theory is reformulated as a particular integral transform.

To summarize, $K(E, \omega)$ can be recovered from $\Pi(\omega)$ by first generalizing $\Pi(\omega)$ to $\Pi(\omega, p, h)$ for a Liouvillian as in Eq. (18) and then carrying out the integrals in

$$
K(E, \omega)=\int_{0}^{\infty} d x \int_{0}^{\infty} d y \oint \frac{d z}{2 \pi i z} \exp (-x-y+z) \Pi\left(E_{+} E_{-}, \frac{x}{z} E_{+},-\frac{y}{z} E_{-}\right) .
$$

As the objects on both sides are formally defined by their diagrammatic series, this is an exact relationship between them. Given a finite set of Liouvillian diagrams, this procedure can clearly be carried out diagram by diagram. On the other hand, the procedure may prove too cumbersome to deal successfully with a particular approximate resummation of Liouvillian perturbation theory. It turns out that our program can be carried through for the $N_{L}=\infty$ limit of MSZ, applied to random matrix ensembles, as we show in Section IV. By contrast, for the quantum Hall problem even the $N_{L}=\infty$ limit requires numerical solution of an integral equation to obtain the Liouvillian. The chore of energy unintegration in this case is much more involved and we will consider it only in a special limit. It is conceivable that in still other cases one may need to resort to numerical resummations of the unintegrated series. 


\section{III. $1 / N_{L}$ EXPANSION}

No exact solution of a problem using the Liouvillian formalism is known at present. It is therefore pertinent to ask whether there are useful, natural approximations in the Liouvillian approach that are hard to uncover in the standard approach. The simplest one, suggested by the interpretation of the Liouvillian as a random Hamiltonian in its own right, is the self-consistent Born approximation (SCBA), employed for this purpose by SMG. In the supersymmetric functional integral approach of MZS, the SCBA is a saddle point approximation, but we will instead take the diagrammatic route, in which it is a summation of all non-crossing diagrams. In either case, the procedure is justified formally by deforming the problem to one with $N_{L}$ Liouvillian flavors and taking the $N_{L}=\infty$ limit. One can then examine the stability of the solution by perturbing in $1 / N_{L}$, in the hope that most of the structure in the problem of interest, $N_{L}=1$, survives to large $N_{L}$.

The recipe for introducing $N_{L}$ flavors into a general Liouvillan problem is as follows. One replicates the original $N^{2}$ dimensional space of bilinears $N_{L}$ times and allows for scattering between different flavors. Then

$$
\Pi_{a A ; b B}^{i j}=\left(a, A ; i\left|\frac{1}{\omega-\hat{\mathcal{L}}+i \delta}\right| b, B ; j\right),
$$

with

$$
\left\langle\hat{\mathcal{L}}^{k l} \hat{\mathcal{L}}^{m n}\right\rangle=\frac{1}{N_{L}}\left(\delta_{k n} \delta_{l m}+\delta_{k m} \delta_{l n}\right)\left\langle\hat{\mathcal{L}}^{k l} \hat{\mathcal{L}}^{k l}\right\rangle .
$$

where in the Liouvillian correlator we have indicated only the flavor indices. The replicated Liouvillian can be thought of as an $N_{L} \times N_{L}$ matrix with individual elements which are themselves $N^{2} \times N^{2}$ Liouvillians of the original, single flavor, problem constructed from different realizations of disorder. Moreover, $\hat{\mathcal{L}}^{k l}=\hat{\mathcal{L}}^{l k}$, so that $\left(N_{L}^{2}+N_{L}\right) / 2$ independent realizations of disorder are necessary to construct a single realization of the replicated problem. The entire $N_{L} N^{2} \times N_{L} N^{2}$ matrix is Hermitian. The construction we have described is, in the terminology of MSZ, the orthogonal generalization of the $N_{L}=1$ problem and is the one they have argued is useful in generating a $1 / N_{L}$ expansion for the localization length exponent for the quantum Hall plateau transition.

To see how the expansion goes, consider as $N_{L} \rightarrow \infty$ the low order diagrams shown in Fig. 5 . Evidently, diagrams with crossed disorder lines are suppressed by factors of $1 / N_{L}$ relative to those without crossed lines. This suppression of crossed diagrams continues in higher orders of the expansion. Thus the leading approximation at large $N_{L}$ is to sum all non-crossing terms, which are of order $N_{L}^{0}$. As usual, this sum can be carried out by solving the equivalent self-consistency equation

$$
\Pi_{a A ; b B}^{i j}=\frac{\delta_{a b} \delta_{A B} \delta_{i j}}{\omega+i \delta}+\frac{1}{\omega+i \delta}[\langle\hat{\mathcal{L}} \cdot \hat{\Pi} \cdot \hat{\mathcal{L}}\rangle \cdot \hat{\Pi}]_{a A ; b B}^{i j}
$$
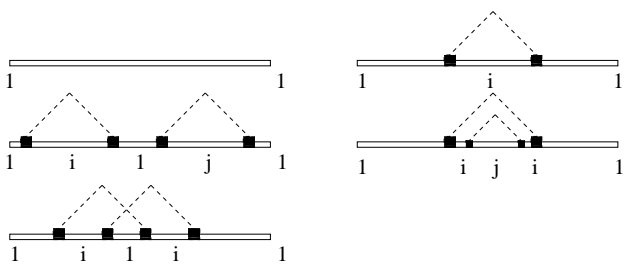

FIG. 5: Liouvillian diagrams up to fourth order in $\hat{\mathcal{L}}$. The last diagram contains a crossing and is smaller by a factor $1 / N_{L}$ than the others, which are of order $N_{L}^{0}$. Only flavor indices are shown explicitly.

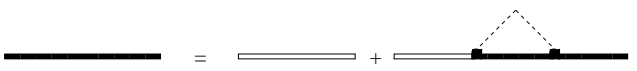

FIG. 6: The Liouvillian SCBA in diagrammatic form. Clear lines represent bare propagators, and filled ones the full propagator, $\hat{\Pi}(\omega)$.

represented graphically in Fig.6. At next order $\left(N_{L}^{-1}\right)$ one needs to sum maximally crossed graphs and rainbow diagrams shown in Fig. 8. At any order the Liouvillian Green function is diagonal in the flavor index

$$
\Pi_{a A ; b B}^{i j}(\omega)=\delta_{i j} \Pi_{a A ; b B}(\omega)
$$

and $\Pi_{a A ; b B}(\omega)$ is taken as an approximation to the $N_{L}=$ 1 Liouvillian Green function.

\section{RANDOM MATRIX THEORY}

We now turn to the problem of random matrix statistics as a test of the Liouvillian large $N_{L}$ approach. The large number of known exact results on this problem and the relative simplicity of calculations make it an ideal test case. While there is no phase transition (all Green functions are analytic inside the band), we will see that there is sufficient non-trivial structure in the statistics of the eigenvalues that can be used diagnostically. Oddly enough, we will find that the Liouvillian analysis will yield some Green functions that are singular inside the band (see Section IVE).

The program is this: for $N \times N$ random matrices, we introduce an enlarged Liouvillian with $N_{L}$ flavors. Now we have (at least) two different limits to consider. The standard one takes $N \gg 1$ at $N_{L}=1$, and leads to a familiar set of simplifications such the Wigner semicircle law for the density of eigenvalues which are summarized below. It also leads to a perturbation theory in $1 / N$ which is the zero dimensional version of impurity averaged perturbation theory for single-particle Green functions in disordered conductors. The new limit we will consider is $N_{L} \gg 1$ at fixed $N$. As in all large- $n$ expansions, this will prove useful if the large $N_{L}$ problem is sufficiently smoothly connected to the $N_{L}=1$ problem of interest. While we will find it useful to then take $N \gg 1$ (but keeping $N \ll N_{L}$ ) as well, the possibility of 
finding non-trivial information on finite matrices is a potential asset of this limit. Similarly, in the application to the quantum Hall plateau transition, the infinite volume limit is taken after $N_{L} \rightarrow \infty$.

Before proceeding we collect some standard definitions and results on the random matrix theory for ease of comparison with the following Liouvillian analysis.

\section{A. Ensembles}

We will be interested in random Hermitean matrices $\hat{H}$ drawn from the Gaussian unitary ensemble (GUE) if $\hat{H}$ is complex, and the Gaussian orthogonal ensemble (GOE) if $\hat{H}$ is required to be purely real. For these ensembles it is sufficient to specifiy the correlators of the matrix elements,

$$
\left\langle H_{a b} H_{c d}\right\rangle=\frac{v^{2}}{N}\left(\delta_{a d} \delta_{b c}+\alpha \delta_{a c} \delta_{b d}\right),
$$

where $\alpha=0$ for GUE and $\alpha=1$ for GOE. The variance has been normalized by the matrix size, $N$, to produce a bandwidth which remains finite as $N \rightarrow \infty$.

\section{B. Correlators}

In addition to the fundamental one and two particle Green functions defined in Section II, we will be inter- ested in the the density of states (DOS) of $\hat{H}$

$$
\rho(E)=\sum_{n} \delta\left(E-\epsilon_{n}\right)=-\frac{1}{\pi} \operatorname{Im} \operatorname{Tr}\left[\frac{1}{E-\hat{H}+i \delta}\right]
$$

and its disorder averaged correlation function

$$
R(E, \omega)=\left\langle\rho\left(E+\frac{\omega}{2}\right) \rho\left(E-\frac{\omega}{2}\right)\right\rangle
$$

$R(E, \omega)$ is related to the two-particle Green functions $K$ by

$$
R(E, \omega)=\frac{1}{4 \pi^{2}} \sum_{a A}\left[K_{a A ; a A}^{+-}+K_{a A ; a A}^{-+}-K_{a A ; a A}^{++}-K_{a A ; a A}^{--}\right]
$$

The Liouvillian Green function allows us to extract the Liouvillian density of states (LDOS)

$$
\Omega(\omega)=\sum_{m n} \delta\left(\omega-\lambda_{m n}\right)=-\frac{1}{\pi} \operatorname{Im} \operatorname{Tr}\left[\frac{1}{\omega-\hat{\mathcal{L}}+i \delta}\right]
$$

and it follows from Eq. (10) that $\Omega(\omega)=\int d E R(E, \omega)$.

Disorder averaging (at any $N$ ) simplifies the structure of various correlators

$$
\begin{aligned}
\left\langle G_{m n}(E)\right\rangle & =\delta_{m n} G(E) \\
\left\langle K_{a A ; b B}(E, \omega)\right\rangle & =\delta_{a b} \delta_{A B} K_{1}(E, \omega)+\delta_{a A} \delta_{b B} K_{2}(E, \omega)+\delta_{a B} \delta_{A b} K_{3}(E, \omega) \\
\left\langle\Pi_{a A ; b B}(\omega)\right\rangle & =\delta_{a b} \delta_{A B} \Pi_{1}(\omega)+\delta_{a A} \delta_{b B} \Pi_{2}(\omega)+\delta_{a B} \delta_{A b} \Pi_{3}(\omega)
\end{aligned}
$$

with the constraints $K_{2}=K_{3}, \Pi_{2}=\Pi_{3}$ for GOE and $K_{3}=\Pi_{3}=0$ for GUE. Finally, the Liouvillian density of states has the form

$$
\Omega(\omega)=-\frac{N^{2}}{\pi}\left(\operatorname{Im} \Pi_{1}(\omega)+\frac{\operatorname{Im}_{2}(\omega)+\operatorname{Im}_{3}(\omega)}{N}\right)(3
$$

where the powers of $N$ arise from taking a trace over the index structure in Eq. (32).

\section{Standard results: large $\mathbf{N}$ at $N_{L}=1$}

At $N=\infty$ the SCBA for the single-particle Green function,

$$
G_{m n}^{\infty}(E)=\delta_{m n} \frac{E}{2 v^{2}}\left(1-\sqrt{1-4 v^{2} / E^{2}}\right)
$$

is exact, and hence the DOS is the Wigner semicircle: $\lim _{N \rightarrow \infty}\langle\rho(E)\rangle / N=(1 / \pi v) \sqrt{1-(E / 2 v)^{2}}$. For $N$ large but finite, the deviations from this limiting form are small. For example, the leading behavior of the DOS at energies above the large $N$ upper band edge, $E=2 v$, is $\rho(E) \sim \exp \left(-4 N(E / v-2)^{3 / 2} / 3\right)$. Higher order correlators of the DOS, including $R(E, \omega)$, can be discussed in each of two limits. First, taking $N \rightarrow \infty$ with energy arguments fixed and all different, they factorize. For example, with $\omega \neq 0$,

$$
R_{\infty}(E, \omega)=\left\langle\rho_{\infty}\left(E+\frac{\omega}{2}\right)\right\rangle\left\langle\rho_{\infty}\left(E-\frac{\omega}{2}\right)\right\rangle .
$$

Second, by scaling the separation of energy arguments with the mean level spacing, universal corrleation functions are obtained, which are dominated at small energy separations by level repulsion. Thus, for $N \omega \ll 1$,

$$
R(E, \omega) \propto(\omega N)^{\beta} \rho(E)^{2},
$$


with $\beta=1$ for GOE and $\beta=2$ for GUE.

Combining these with our earlier listing of the general properties of Liouvillians we conclude that the exact LDOS will have almost all its weight within the range $-4 v<\omega<4 v$ for $N \gg 1$. For $N=\infty$ it is exactly zero outside and vanishes with zero slope (quadratically $\left.\sim(4 v \pm \omega)^{2}\right)$ near the edges. Finally, at finite $N$, there should be a pseudogap of width $v / N$ near $\omega=0$ with details depending on the symmetry of the ensemble.

\section{RMT Liouvillian}

$$
\text { 1. } N_{L}=\infty, N \text { arbitrary }
$$

For the GUE Eq. (25) becomes

$$
\Pi_{1}(\omega)=\frac{1}{\omega+i \delta}+\frac{2 v^{2} \Pi_{1}(\omega)^{2}}{\omega+i \delta}
$$

$$
\begin{aligned}
& \Pi_{2}(\omega)=-\frac{2 v^{2}}{N(\omega+i \delta)} \Pi_{1}(\omega)^{2}=\frac{1}{N}\left(\frac{1}{\omega+i \delta}-\Pi_{1}(\omega)\right) \\
& \Pi_{3}(\omega)=0
\end{aligned}
$$

The solution is similar to that for $N=\infty$ RMT (cf. Eq. 34). Using Eq. (33) the LDOS is

$$
\Omega(\omega)=\delta(\omega)+\frac{N^{2}-1}{\pi \sqrt{2 v^{2}}} \sqrt{1-\frac{\omega^{2}}{8 v^{2}}}
$$

for $|\omega|<2 \sqrt{2} v$ and zero otherwise. Notice that the support of the spectrum at $N_{L}=\infty$ is completely independent of $N$.

Turning to GOE, the self-consistency condition reduces to the quadratic equations

$$
\begin{aligned}
& \Pi_{1}(\omega)=\frac{1}{\omega+i \delta}+\frac{2 v^{2}(N+1)}{N(\omega+i \delta)}\left(\Pi_{1}(\omega)^{2}-\Pi_{3}(\omega)^{2}\right) \\
& \Pi_{3}(\omega)=\Pi_{2}(\omega)=\frac{2 v^{2}}{N(\omega+i \delta)}\left(\Pi_{3}(\omega)^{2}-\Pi_{1}(\omega)^{2}\right)=\frac{1}{N+1}\left(\frac{1}{\omega+i \delta}-\Pi_{1}(\omega)\right)
\end{aligned}
$$

with solution

$$
\Pi_{1}(\omega)=\frac{-4 v^{2}+(1+N)\left(N(\omega+i \delta)^{2}-\sqrt{16 v^{2}-8 N(1+N) v^{2}(\omega+i \delta)^{2}+N^{2}(\omega+i \delta)^{4}}\right)}{4 N(2+N) v^{2}(\omega+i \delta)},
$$

leading to the LDOS

$$
\Omega(\omega)=-\frac{1}{\pi}\left(N^{2}-\frac{2 N}{N+1}\right) \operatorname{Im} \Pi_{1}(\omega)-\frac{2 N}{\pi(N+1)} \operatorname{Im} \frac{1}{\omega+i \delta} .
$$

As $N \rightarrow \infty$ the leading, $\mathcal{O}\left(N^{2}\right)$, piece of this result is identical to the corresponding piece of $\Omega(\omega)$ for the GUE. For finite $\mathrm{N}$, however, the LDOS is zero in the window $|\omega|<\sqrt{2 v^{2}} / N$, in stark contrast with the GUE result (or anything else one might expect of a disordered system: according to this approximation the spectrum of the disordered problem is completely gapped). These results are illustrated in Fig. 7 .

There are at least four important differences between these exact $N_{L}=\infty$ results and the corresponding, correct, $N_{L}=1$ solutions:

- At $N_{L}=\infty$ results for both GUE and GOE imply a bandwidth of $2 \sqrt{2} v$ for the underlying random matrices. This is a factor of $\sqrt{2}$ less than that of the correct solution.

- The infinite gradient of the $N_{L}=\infty$ expression for $\Omega(\omega)$ at the Liouvillian band edges is at odds with the exact result, which has vanishing slope.
Contributions to the LDOS at the Liouvillian band edges come from pairs of random matrix eigenvalues which are near opposite random matrix band edges. If one assumes that $R$ will factorize in such cases, which is certainly true of the exact result and should be expected anyway for such widely separated eigenvalues, the form found for LDOS implies a DOS divergent near the random matrix band edges, with $\rho(E) \sim(v \sqrt{2} \pm E)^{-1 / 4}$. In fact, we shall see that $R$ does not factorize at $N_{L}=\infty$, which turns out to be the central pathology of that limit.

- Although there is a delta function at zero frequency, arising from zero eigenvalues of $\hat{\mathcal{L}}$ (see Eq. (10)), its weight is 1 , and not $N$ as it should be.

- Finally, the two solutions are qualitatively wrong in the small frequency limit $(|\omega| \lesssim 1 / N)$ in opposite 


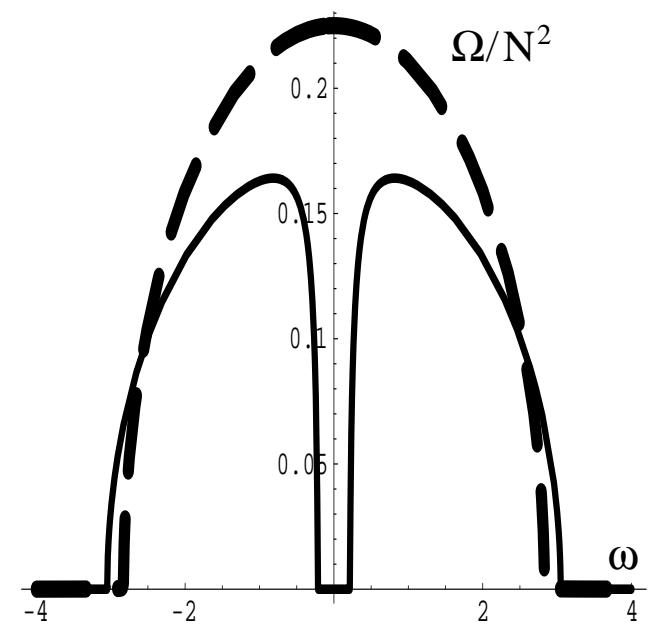

FIG. 7: LDOS scaled by $N^{2}$ at $N_{L}=\infty$ with disorder strength $v=1$. Solid line is GOE (Eq. 41) at $N=6$ while the broken one is GOE (Eq. 41) at $N=\infty$ and GUE (Eq. 38) at arbitrary $N$. The delta function at zero frequency has been suppressed.

ways. The GUE LDOS is non-zero with no indication of level repulsion, while the GOE develops a clean gap over a region of $\mathcal{O}(1 / N)$.

The $N_{L}=\infty$ result above is clearly not an adequate approximation to $N_{L}=1$ even for the purposes of computing properties of the Liouvillian. In the next section we will see that matters get worse when we energy unintegrate the $N_{L}=\infty$ result. Before turning to that, we first ask whether one may construct a smooth interpolation between the two limits by perturbing in $1 / N_{L}$. We offer evidence that this is unlikely, both from considering the $1 / N_{L}$ expansion analytically, and from studying the problem numerically for a range of $N_{L}$ values.

\section{Leading $1 / N_{L}$ corrections}

As discussed in Section III, the leading correction for large $N_{L}$ to the $N_{L}=\infty$ Liouvillian Green function is given by the maximally crossed diagrams. These are resummed by two consecutive geometric series, represented graphically in Fig. 8.

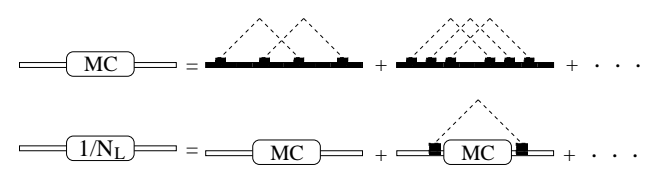

FIG. 8: All diagrams for $\hat{\Pi}(\omega)$ at $\mathcal{O}\left(1 / N_{L}\right)$.

Denoting the $N_{L}=\infty$ solution by $\Pi_{1}$, the result of this calculation for the GUE is

$$
\delta \Omega(\omega)=\frac{1-N^{2}}{N_{L} \pi} \operatorname{Im}\left(\frac{2\left(\Pi_{1}\right)^{5} v^{4}}{\left(1-v^{2}\left(\Pi_{1}\right)^{2}\right)\left(1-2 v^{2}\left(\Pi_{1}\right)^{2}\right)}\right)
$$

As is the case with $1 / N$ corrections to the RMT DOS,

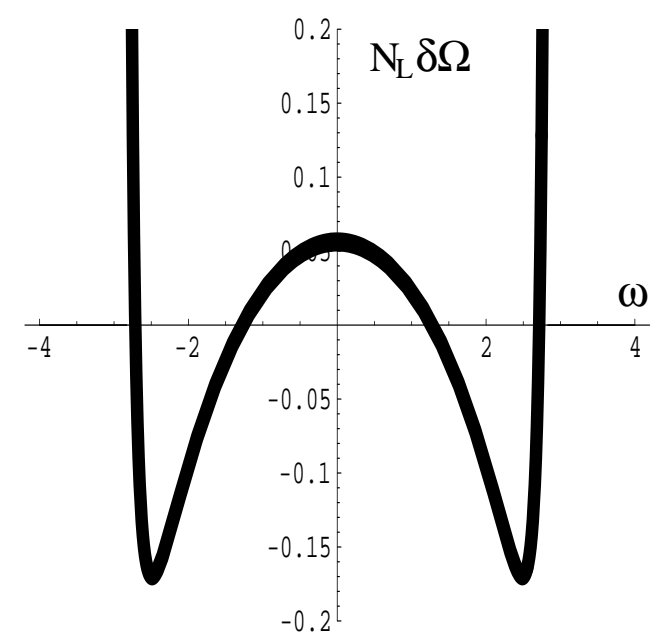

FIG. 9: $1 / N_{L}$ correction to the LDOS for GUE

the $1 / N_{L}$ correction to the LDOS is finite near $\omega=0$ and develops a singularity at band edges, as illustrated in Fig. 9. In the case of the GOE, the $1 / N_{L}$ correction, which for brevity we do not display, is divergent at all four band edges. These divergences show that any attempt to fix the bandwidth problems of the $N_{L}=\infty$ limit, if feasible, must involve an analysis including contributions at all orders in $1 / N_{L}$. We have not pursued this analysis further. Instead, we offer evidence from numerical studies that expansion about $N_{L}=\infty$ is unlikely to yield useful information on behavior at $N_{L}=1$.

\section{Numerics at small $N_{L}$}

The simplicity of the random matrix problem affords us a different, more direct line of attack via exact diagonalization. Specifically, we have diagonalised numerically Liouvillian RMT for a range of $N_{L}$ and $N$, both to test the relevance of analytic results above and also to search for new features, especially in the interesting regime of small $N_{L}$. Representative plots of $\Omega(\omega)$ are shown for the GUE in Fig. 10 and for the GOE in Fig. 11, both covering a range in $N_{L}$ at $N=2$. We have searched for, but found no significant qualitative differences at larger $\mathrm{N}$. Our conclusions from these figures are as follows. First, the gross of features of the $N_{L}=\infty$ limit (see Fig. 7) are already observable at $N_{L}$ as small as $N_{L}=8$. Second, Liouvillian theories at $N_{L}>1$ are sufficiently different from the theory at $N_{L}=1$, even for $N_{L}=2$ and 3 , that the quantitative utility of any $1 / N_{L}$ corrections seems questionable. Readers should note especially the rapid change in the distribution of small eigenvalues as $N_{L}$ is increased. Third, while one clearly cannot hope to investigate analyticity conclusively by these means, there are two features to the numerical results which suggest behavior non-analytic in $1 / N_{L}$ at $N_{L}=\infty$. One of these 
is the number of states outside the $N_{L}=\infty$ band, which appears to decrease exponentially in $N_{L}$, as in the band tails of the RMT DOS. The other is the presence of oscil- lations in the LDOS for the GUE at small energies, as a function of $N_{L}: \Omega(0)$ vanishes for odd $N_{L}$ and is non-zero for even ones.

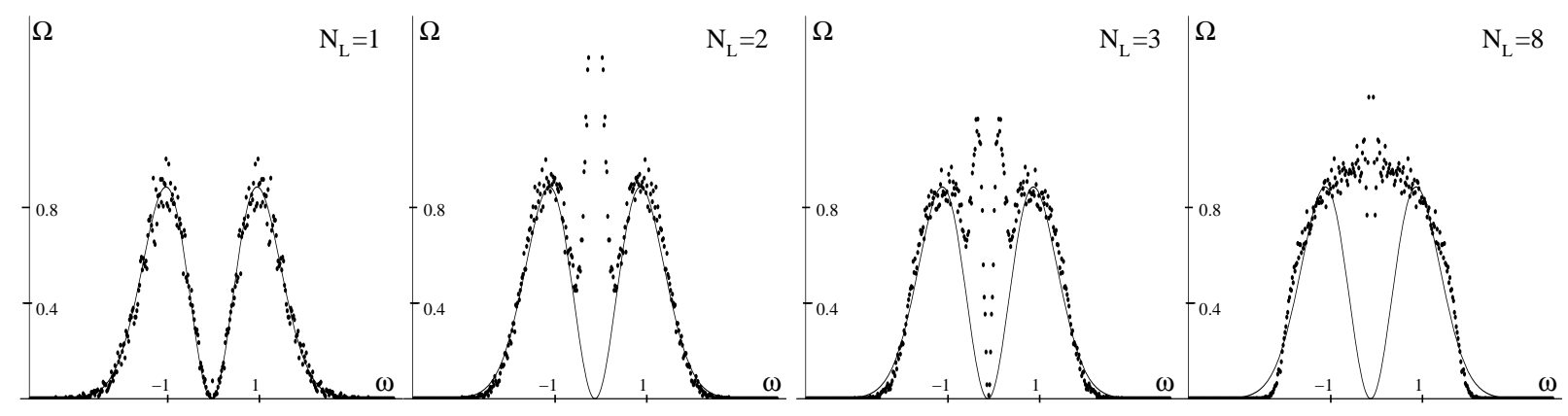

FIG. 10: Evolution of $\Omega(\omega)$ from $N_{L}=1$ to $N_{L}=8$ for $\mathrm{N}=2$ GUE of random matrices. Solid line is the exact solution for $N_{L}=19$. Delta functions at zero frequency have been suppressed.

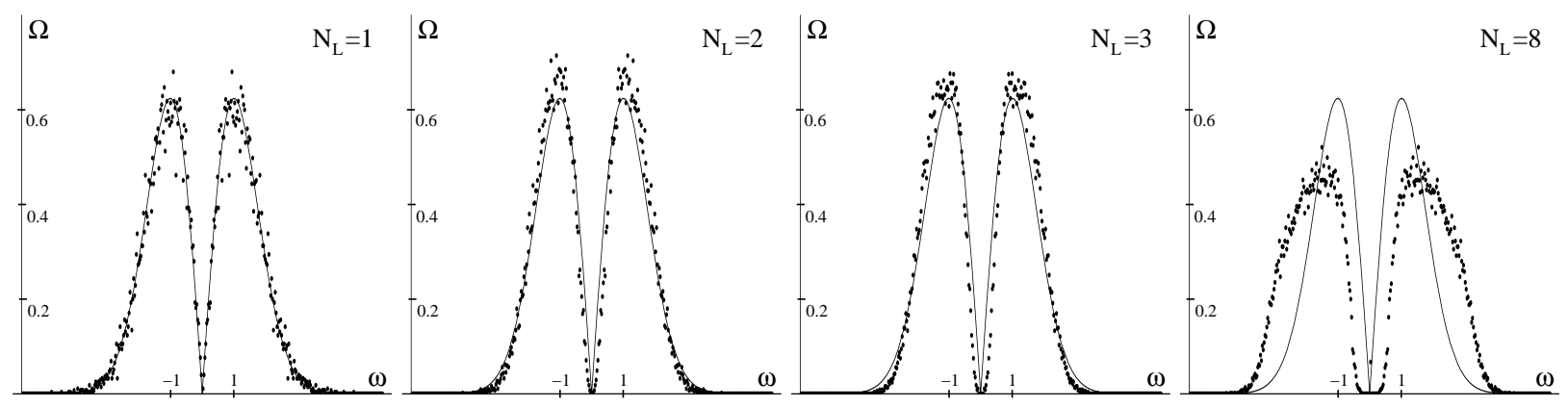

FIG. 11 Evolution of $\Omega(\omega)$ from $N_{L}=1$ to $N_{L}=8$ for $\mathrm{N}=2$ GOE of random matrices. Solid line is the exact solution for $N_{L}=1 \mathrm{1}$. Delta functions at zero frequency have been suppressed.

\section{E. Undoing the energy integration}

In this subsection we apply the formalism developed in Sec IIE to translate the $N_{L}=\infty$ RMT calculation into Green function language. We start from Eq. (18), applied to RMT. Summing the non-crossed diagrams with $\hat{\mathcal{L}}(p, h)$ leads for the GUE, to the self-consistency equations, modified from Eq. (37) to

$$
\begin{aligned}
& \Pi_{1}(\omega)=\frac{1}{\omega+i \delta}+\frac{\Pi_{1}(\omega) v^{2}}{\omega+i \delta}\left[\left(p^{2}+h^{2}\right) \Pi_{1}(\omega)+\frac{(p-h)^{2}}{N} \Pi_{2}(\omega)\right] \\
& \Pi_{2}(\omega)=-\frac{2 p h v^{2} \Pi_{1}(\omega)^{2}}{N(\omega+i \delta)}+\frac{v^{2}(p-h)^{2} \Pi_{2}(\omega)}{\omega+i \delta}\left[\Pi_{1}(\omega)+\frac{\Pi_{2}(\omega)}{N}\right] \\
& \Pi_{3}(\omega)=0 .
\end{aligned}
$$

We now take the further limit $N \rightarrow \infty$ whereon the solution is

$$
\Pi_{1}(\omega)=\frac{\omega}{2 v^{2}\left(p^{2}+h^{2}\right)}\left(1-\sqrt{1-\frac{4\left(p^{2}+h^{2}\right) v^{2}}{(\omega+i \delta)^{2}}}\right)
$$

In this limit the solution for the GOE is identical.

Using Eq. 22) we obtain $K_{a A ; b B}^{+-}(E, \omega)=$ $\delta_{a b} \delta_{A B} K_{1}(E, \omega)$ where 


$$
K_{1}(E, \omega)=\int_{0}^{\infty} d x \int_{0}^{\infty} d y \exp (-x-y) \oint \frac{d z e^{z} E_{+}^{-1} E_{-}^{-1}}{\left.2 \pi i 2 v^{2}\left[\left(x E_{-}^{-1}\right)^{2}+\left(y E_{+}^{-1}\right)^{2}\right)\right]}\left[z-\sqrt{z^{2}-4 v^{2}\left[\left(x E_{+}^{-1}\right)^{2}+\left(y E_{-}^{-1}\right)^{2}\right]}\right] .
$$

We carry out the integral for large positive $E_{ \pm}$(see Appendix B for details) and obtain results for general $E_{ \pm}$by analytic continuation. We find

$$
K_{1}\left(E_{+}, E_{-}\right)=\frac{1}{2 v^{2}}\left[\sin ^{-1}\left(\frac{E_{+} \sqrt{E_{+}^{2}-4 v^{2}}+E_{-} \sqrt{E_{-}^{2}-4 v^{2}}}{E_{+}^{2}+E_{-}^{2}-4 v^{2}}\right)-\frac{\pi}{2}\right]
$$

where the signs of the square roots are positive for real $E_{ \pm}>2 v$, and branch cuts in the complex $E_{+}$and $E_{-}$planes run on the real axes, from $E_{ \pm}=-2 v$ to $E_{ \pm}=2 v$.

The very first thing to note about this result is that, unlike the exact answer

$$
K_{a A ; b B}^{+-}=G^{+}\left(E_{+}\right) G^{-}(E-)=\delta_{a b} \delta_{A B} \frac{E_{+} E_{-}}{4 v^{4}}\left(1-\sqrt{1-\frac{4 v^{2}}{E_{+}^{2}}}\right)\left(1-\sqrt{1-\frac{4 v^{2}}{E_{-}^{2}}}\right),
$$

it does not factor in its dependence upon $E_{ \pm}$. At $N=\infty$, where the energies $E_{ \pm}$involve eigenvalues which are at infinite separation on the scale of the level spacing, this is manifestly wrong.

To proceed further in this discussion, we need to examine the analytic structure of Eq. (44). Observe that the result is an analytic function of real $E_{+}$and $E_{-}$when both $E_{+}>2$ and $E_{-}>2$. In this region it is the sum of the convergent perturbative expansion in inverse powers of $E_{ \pm}$that is generated by unintegrating the $N_{L}=\infty$ series term by term. Also, in this region $K^{+-}$is purely real and the choice of the sign of the imaginary infinitesimals in $E_{ \pm}$is immaterial: it equally well describes an approximation to $K^{++}$.

The series diverges on approaching the boundary of this region. In the two-dimensional plane of real $E_{+}, E_{-}$, this happens at the outer boundary of the cross-shape formed by the four lines $E_{+}= \pm 2 v$ and $E_{-}= \pm 2 v$, shown in Fig.12. Inside this cross-shape, the choice of infinitesimal imaginary parts to $E_{ \pm}$is essential to specify which side one is of the branch cuts in Eq. (44). De- pending on how this is done, we obtain inside the cross both $K^{+-}$and $K^{++}$, which are no longer equal but are both generically complex. The development of imaginary parts to the Green functions occurs when one of their arguments enters the band. Indeed, in the exact expression, Eq. (45), this is trivially true.

Random matrices do not exhibit a phase transition and so we should expect that $K^{+-}$and $K^{++}$are analytic functions of $E_{+}$and $E_{-}$except at the band edges, $E_{ \pm}^{2}=4 v^{2}$. This is true of the exact result, Eq. (45). Surprisingly, it is not true of the correlators derived from Eq. (44). Specifically, inside the cross, while $K^{+-}$is analytic, $K^{++}$exhibits singularities on the circle $E_{+}^{2}+E_{-}^{2}=$ $4 v^{2}$. For example, along the line $E_{+}=E_{-}$, i.e. $\omega=0$, we find

$$
K^{+-}(E, \omega=0)=\frac{\pi}{4 v^{2}}
$$

while

$$
\operatorname{Re} K^{++}(E, \omega=0)=\frac{\pi}{4 v^{2}} \operatorname{sgn}\left(\frac{E}{v}-\sqrt{2}\right)
$$

for $0<E / v<2$ and

$$
\operatorname{Im} K^{++}(E, \omega=0)=\left\{\begin{array}{ccc}
\frac{1}{v^{2}} \cosh ^{-1}\left(\frac{E / v}{\sqrt{2(E / v)^{2}-4}}\right) \text { when } & \sqrt{2}<E / v<2 \\
-\frac{1}{v^{2}} \sinh ^{-1}\left(\frac{E / v}{\sqrt{4-2(E / v)^{2}}}\right) & \text { when } & 0<E / v<\sqrt{2}
\end{array}\right.
$$

where the negative branch is picked for the $\cosh ^{-1}$. These results are plotted in Fig. 13 and Fig. 14 along with the exact Green functions for comparison. 
Finally, one can extract from the knowledge of both $K^{+-}$and $K^{++}$the correlator of the density of states, which has the simple form

$$
R(E, \omega)=\frac{1}{4 \pi v^{2}} \Theta\left(4 v^{2}-2 E^{2}-\frac{\omega^{2}}{2}\right)
$$

It is constant inside the circle $E_{+}^{2}+E_{-}^{2}=4 v^{2}$ and zero outside. This solves one remaining puzzle. While the $\mathrm{Li}$ ouvillian derived two particle Green functions imply the same RMT band as the the exact ones, in the sense that both are analytic outside the cross, we noted previously that the Liouvillian bandwidth was wrong by a factor of $\sqrt{2}$. We can now recover the LDOS, Eq. (38), by integrating $R(E, \omega)$ over energy

$\frac{1}{N^{2}} \Omega(\omega)=\int \frac{d E}{4 \pi v^{2}} \Theta\left(2 E^{2}+\frac{\omega^{2}}{2}-4 v^{2}\right)=\frac{1}{\pi \sqrt{2 v^{2}}} \sqrt{1-\frac{\omega^{2}}{8 v^{2}}}$,

which serves also to verify that our analysis is internally consistent.

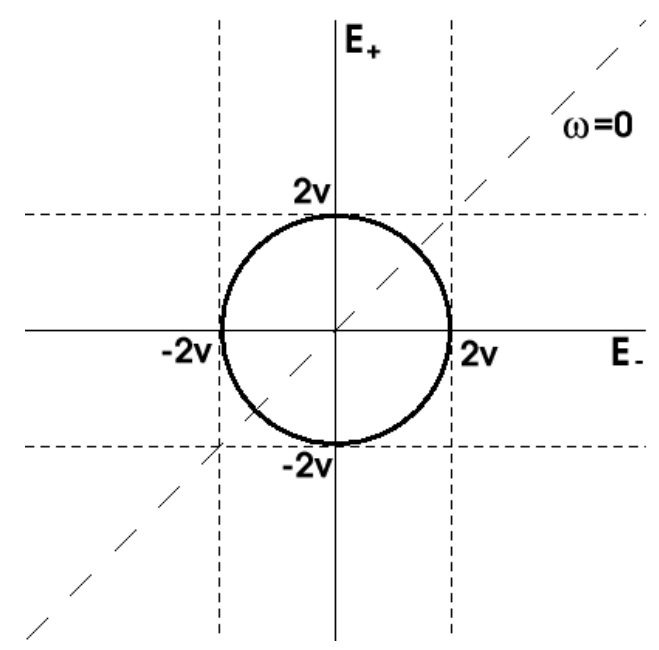

FIG. 12: Analytic structure of $K\left(E_{+}, E_{-}\right) . R(E, \omega)$ is finite inside the circle.

\section{F. Summary of random matrix analysis}

Briefly, in our direct examination of the Liouvillian method we find that the large $N_{L}$ limit is unrepresentative of the behavior of the $N_{L}=1$ problem. Upon energy unintegrating the Liouvillian $N_{L}=\infty$ calculation, we find a pathological approximation which fails to factor correctly and even produces singularities inside the band. Together, these facts indicate that the Liouvillian $1 / N_{L}$ expansion is not a useful way of approaching the random matrix problem.

In this analysis we have utilized two complementary perspectives on the large $N_{L}$ method and a comment on those is perhaps useful. The first perspective is that

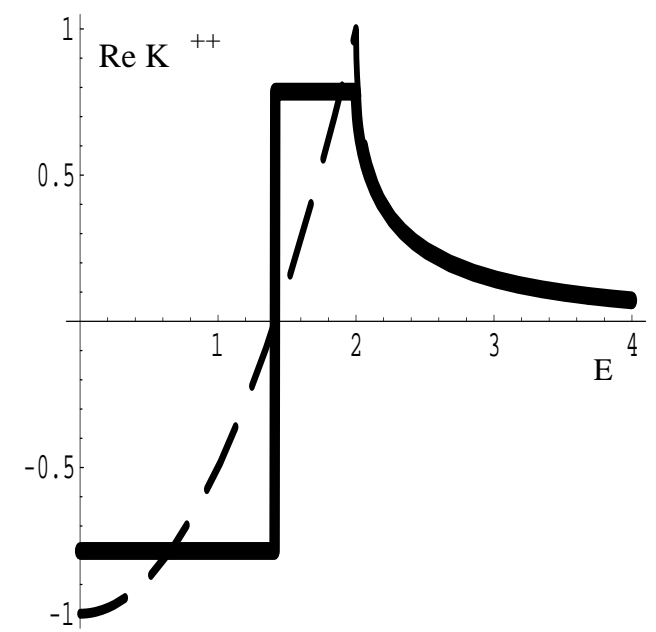

FIG. 13: Real part of $K^{++}(E, E)$ corresponding to the energy resolved Liouvillian solution (solid line) vs. exact result (dashed line).

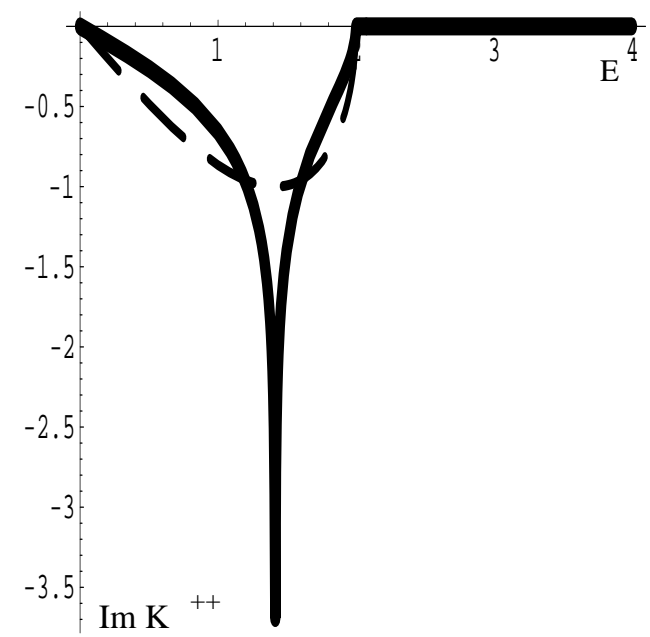

FIG. 14: Imaginary part of $K^{++}(E, E)$ corresponding to the energy resolved Liouvillian solution (solid line) vs. exact result (dashed line).

we generate a family of problems with enlarged symmetry groups indexed by an integer each one of which can be studied directly - which is what we have done in the numerical analysis. The enlarged problems are defined by multi-flavor Liouvillians that do not themselves arise from from single particle Hamiltonians. The second perspective treats the large $N_{L}$ limit as formalizing perturbation theory about a saddle point that is believed to capture the relevant physics. In this fashion we generate a series in powers of $1 / N_{L}$ whose first term comes solely from the saddle point. The utility of the method is, of course, that setting $N_{L}=1$ in the perturbation theory yields the answer for the case of interest. In using the $N_{L}=\infty$ answer as input for the energy unintegration algorithm we have followed standard practice and simply truncated the series at its first term and set $N_{L}=1$. If 
the large $N_{L}$ method is useful in describing the $N_{L}=1$ problem this should be a sensible approximation. Since we find that this is not so, the premise must be flawed.

\section{THE QUANTUM HALL TRANSITION}

Our Liouvillian analysis of the random matrix problem suggests to us that the method is unreliable and hence not to be trusted in its application to the quantum Hall problem. This is a disincentive to pursue a similarly detailed study of the quantum Hall case, especially as it would be a substantial undertaking: even the Liouvillian $N_{L}=\infty$ analysis of that problem requires a numerical solution, and a full energy unintegration would require much more intensive numerical work. We therefore confine ourselves to three sets of observations. First, we confirm that our general Liouvillian formalism reduces to the one used by SMG and MSZ for the quantum Hall case. Second, we exhibit the unintegration algorithm specialized to this case. Third, we apply the unintegration algorithm to a special sector of the $N_{L}=\infty$ problem, that of very large momenta, where it reduces to precisely the Liouvillian random matrix problem studied in the last section. This then imbeds the pathologies of that problem into the quantum Hall case.

\section{A. Liouvillian formulation}

We consider a charged particle moving in a magnetic field on a torus of area $2 \pi l^{2} N$, where $l \equiv(\hbar c / e B)^{1 / 2}$ is the magnetic length and $N$ is the integer degeneracy of each Landau level. We denote the projection operator onto the lowest Landau level by $\mathcal{P}$. As the kinetic energy is constant within each Landau level, the Hamiltonian projected to the lowest Landau level is simply $H=\mathcal{P} V(\mathbf{r}) \mathcal{P}$ where $V(\mathbf{r})$ is the impurity potential.

It is convenient to work initially with the states $\{\mathcal{P}|\mathbf{r}\rangle\}$ which form an overcomplete basis for the lowest Landau level; to lighten notation we will write $\{|\mathbf{r}\rangle\}$ for $\{\mathcal{P}|\mathbf{r}\rangle\}$. In terms of these we can define the single-particle Green functions in real space

$$
G^{ \pm}\left(E ; \mathbf{r}_{1}, \mathbf{r}_{2}\right)=\left\langle\mathbf{r}_{1}\left|\frac{1}{(E-H \pm i \delta)}\right| \mathbf{r}_{2}\right\rangle
$$

and the two-particle retarded-advanced Green function in real space

$$
K^{+-}\left(E, \omega ; \mathbf{r}_{1}, \mathbf{r}_{2}\right)=G^{-}\left(E-\omega / 2 ; \mathbf{r}_{1}, \mathbf{r}_{2}\right) G^{+}\left(E+\omega / 2 ; \mathbf{r}_{2}, \mathbf{r}_{1}\right)
$$

where we have chosen a pairing of position coordinates appropriate for a diffusion propagator. These are evidently the analogs of Eqns. (1) and (2) in Section II. From these we derive the analog of Eq. (3),

$$
\Pi\left(\mathbf{r}_{\mathbf{1}}, \mathbf{r}_{\mathbf{2}}, \omega\right)=\int_{-\infty}^{\infty} \frac{d E}{2 \pi i} K^{+-}\left(E, \omega ; \mathbf{r}_{\mathbf{1}}, \mathbf{r}_{\mathbf{2}}\right)
$$

$$
=\left(\mathbf{r}_{1}, \mathbf{r}_{1}\left|\frac{1}{(\omega+i \delta-\mathcal{L})}\right| \mathbf{r}_{2}, \mathbf{r}_{2}\right) \text {. }
$$

As disorder averaging restores translational invariance, we take Fourier transforms and define (denoting the disorder averaged quantities by the same symbols),

$$
\begin{gathered}
K^{+-}(E, \omega ; \mathbf{q})= \\
\frac{1}{2 \pi l^{2} N} \iint K^{+-}\left(E, \omega ; \mathbf{r}_{1}, \mathbf{r}_{2}\right) e^{i \mathbf{q} \cdot\left(\mathbf{r}_{2}-\mathbf{r}_{1}\right)} d \mathbf{r}_{1} d \mathbf{r}_{2} \\
\Pi(\omega ; \mathbf{q})=\frac{1}{2 \pi l^{2} N} \iint \Pi\left(\omega ; \mathbf{r}_{1}, \mathbf{r}_{2}\right) e^{i \mathbf{q} \cdot\left(\mathbf{r}_{2}-\mathbf{r}_{1}\right)} d \mathbf{r}_{1} d \mathbf{r}_{2}
\end{gathered}
$$

and the states

$$
\left.\left.\mid \rho_{\mathbf{q}}\right)=\int \mid \mathbf{r}, \mathbf{r}\right) \exp (-i \mathbf{q . r}) d^{2} \mathbf{r},
$$

which represent the projected density operators $\mathcal{P} \exp (-i \mathbf{q . r}) \mathcal{P}$ in the space of operators $\mathcal{A}$ on the lowest Landau level. In terms of these we can rewrite Eq. (53) as

$$
\begin{aligned}
\Pi(\mathbf{q}, \omega) & =\int_{-\infty}^{\infty} \frac{d E}{2 \pi i} K^{+-}(E, \omega ; \mathbf{q}) \\
& =\frac{1}{2 \pi \hbar l^{2} N}\left\langle\left(\rho_{\mathbf{q}}\left|\frac{1}{(\omega+i \delta-\mathcal{L})}\right| \rho_{\mathbf{q}}\right)\right\rangle .
\end{aligned}
$$

In place of the density operators, it is slightly more convenient to use (as a basis for $\mathcal{A}$ ) the magnetic translation operators $\tau_{\mathbf{q}}=e^{+l^{2} q^{2} / 4} \rho_{\mathbf{q}}$, which are closed under the algebra

$$
\tau_{\mathbf{q}} \tau_{\mathbf{q}^{\prime}}=\exp \left(i l^{2} \mathbf{q} \wedge \mathbf{q}^{\prime} / 2\right) \tau_{\mathbf{q}+\mathbf{q}^{\prime}}
$$

where $q \wedge q^{\prime}=\epsilon_{i j} q_{i} q_{j}^{\prime}$, and are orthogonal with normalisation

$$
\left(\tau_{\mathbf{q} 1} \mid \tau_{\mathbf{q} 2}\right)=N \delta_{\mathbf{q} 1, \mathbf{q} 2} .
$$

With the identification

$$
\left.\left.\mid \rho_{\mathbf{q}}\right)=e^{-l^{2} q^{2} / 4} \mid \tau_{\mathbf{q}}\right)
$$

one can show that in this basis the matrix elements of $\hat{\mathcal{L}}=\hat{H} \circ \hat{1}-\hat{1} \circ \hat{H}$ are

$$
\begin{aligned}
\mathcal{L}_{\mathbf{q q}^{\prime}} & =V_{\mathbf{q}-\mathbf{q}^{\prime}} e^{-\frac{\left|\mathbf{q}-\mathbf{q}^{\prime}\right|^{2} l^{2}}{2}}\left[e^{i \frac{\mathbf{q} \wedge \mathbf{q}^{\prime} l^{2}}{2}}-e^{-i \frac{\mathbf{q} \wedge \mathbf{q}^{\prime} l^{2}}{2}}\right] \\
& =2 i V_{\mathbf{q}-\mathbf{q}^{\prime}} e^{-\frac{\left|\mathbf{q}-\mathbf{q}^{\prime}\right|^{2} l^{2}}{2}} \sin \left(\frac{\mathbf{q} \wedge \mathbf{q}^{\prime} l^{2}}{2}\right) .
\end{aligned}
$$

With these we arrive finally at the form

$$
\Pi(\mathbf{q}, \omega)=\frac{\exp \left(-q^{2} l^{2} / 2\right)}{2 \pi l^{2}}\left\langle\left[\frac{1}{\omega+i \delta-\hat{\mathcal{L}}}\right]_{\mathbf{q q}}\right\rangle
$$

which is the one introduced by SMG (up to a constant due to difference in definitons). 
From known facts about the two particle Green function it can be deduced (see Appendix A for details) that the exact Liouvillian self energy varies for small $q, \omega$ as

$$
\omega-\Pi^{-1}(\mathbf{q}, \omega) \equiv i q^{2} D(\omega) \sim i q^{2} \omega^{\frac{1}{2 \nu}} .
$$

This is sub-diffusive behavior and is controlled by the correlation length exponent as $q \rightarrow 0$ at fixed $\omega$. The combined work of SMG and MSZ has yielded a self energy, in the $1 / N_{L}$ expansion, of this form with

$$
D(\omega) \sim D_{0}\left[1+O\left(\frac{\log \omega}{N_{L}}\right)\right],
$$

suggestive of an expansion of a critical power law. In the following subsection we note that the general algorithm of Section II could be used to examine this result at different energies in the disorder broadened Landau level.

\section{B. Energy unintegration}

Formally, the procedure for recovering $K(\mathbf{q}, E, \omega)$ from $\Pi(\mathbf{q}, \omega)$ is a straightforward specialization of Section $\Pi$. One starts by deforming $\hat{\mathcal{L}}$ to

$$
\mathcal{L}_{\mathbf{q} \mathbf{q}^{\prime}}(p, h)=V_{\mathbf{q}-\mathbf{q}^{\prime}} e^{-\frac{\left|\mathbf{q}-\mathbf{q}^{\prime}\right|^{2} l^{2}}{2}}\left[p e^{i \frac{\mathbf{q} \wedge \mathbf{q}^{\prime}}{2} l^{2}}-h e^{-i \frac{\mathbf{q} \wedge \mathbf{q}^{\prime}}{2} l^{2}}\right] .
$$

This generates a deformation of any given Liouvillian diagram or of some partial summation that leads to an approximate form for $\Pi(\mathbf{q}, \omega)$. In either case, carrying out the replacements in Eq. (18) and the integrals in Eq. (22) gives the corresponding energy resolved expressions.

As for the general example of Sec. II the algorithm relies on disorder contractions to keep track of the topology of individual diagrams with $p$ and $h$ used to assign appropriate weights via Eq. 22. Since the first step of the procedure is particularly transparent in the LL setting we briefly consider the analog of Fig. 3. In particular, for each of the ten "unfolded" diagrams we can immdediately write down its value by drawing momentum carrying lines (both disorder and external) inside the "particle-hole circle" and integrating correlators of disorder potential with an exponential/phase factor chosen according to diagram's topology. For example, the diagram of Fig. 15 is given (aside from energy dependence) by

$e^{-\frac{Q^{2} l^{2}}{2}} \int \frac{d^{2} q d^{2} p}{(2 \pi)^{4}}\left\langle V_{\mathbf{q}} V_{-\mathbf{q}}\right\rangle\left\langle V_{\mathbf{p}} V_{-\mathbf{p}}\right\rangle e^{-\frac{\left(p^{2}+q^{2}\right) l^{2}}{2}-i(\mathbf{q} \wedge \mathbf{p}+\mathbf{p} \wedge \mathbf{Q}) l^{2}}$,

and boils down to evaluating a determinant. The wedge factors $(\mathbf{q} \wedge \mathbf{p} \equiv \hat{\mathbf{z}} \cdot \mathbf{q} \times \mathbf{p})$ appear whenever two momentum lines cross (one also selects a sign convention) and effectively serve as a fingerprint of each diagram. These diagrammatic rules are familiar to workers in the field and have enabler_considerable progress in series analysis of the problem 15 16. Here, they illustrate the connection

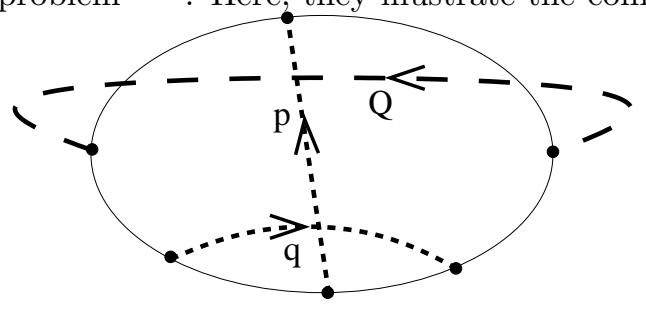

FIG. 15: One of the ten diagrams Fig. 3 redrawn and relabelled using momentum coordinates.

between Liouvillian and Green function perturbation series.

In order to apply this procedure in full to the Liouvillian $N_{L}=\infty$ limit, we must compute $\Pi(\mathbf{q}, \omega, p, h)$, which in turn requires numerical solution of an integral equation and is therefore a substantial undertaking. We turn now to the large $\mathbf{q}$ limit, for which the energy unintegration can be carried out more easily.

\section{Energy unintegration at $N_{L}=\infty$, as $\mathbf{q} \rightarrow \infty$}

The key observation $\mathrm{B}$ that allows us to execute the energy unintegration procedure in this limit is that the self-consistency equation simplifies drastically. Defining $\bar{\Pi}(\mathbf{q}, \omega)$ by $\Pi(\mathbf{q}, \omega)=e^{-l^{2} q^{2} / 2} \bar{\Pi}(\mathbf{q}, \omega)$, as $q \rightarrow \infty$ the selfconsistency equation

$$
\bar{\Pi}(\mathbf{q}, \omega)=\frac{1}{\omega+i \delta}+\frac{\bar{\Pi}(\mathbf{q}, \omega)}{\omega+i \delta} \int \frac{d^{2} q^{\prime}}{4 \pi^{2}}\left\langle\hat{\mathcal{L}}_{\mathbf{q}, \mathbf{q}+\mathbf{q}^{\prime}} \hat{\mathcal{L}}_{\mathbf{q}+\mathbf{q}^{\prime}, \mathbf{q}}\right\rangle \bar{\Pi}\left(\mathbf{q}+\mathbf{q}^{\prime}, \omega\right)
$$

reduces to

$$
\bar{\Pi}(\infty, \omega)=\frac{1}{\omega+i \delta}+\frac{2 v^{2} \bar{\Pi}^{2}(\infty, \omega)}{\omega+i \delta},
$$

which is identical to the corresponding RMT equation (either GUE or GOE) at $N=\infty$. It follows then that the energy unintegrated solution of this equation, the quantity $\bar{K}\left(\infty, E_{+}, E_{-}\right)=\lim _{\mathbf{q} \rightarrow \infty} e^{+q^{2} l^{2} / 2} K\left(\mathbf{q}, E_{+}, E_{-}\right)$, is given by the expression already exhibited in Eq. (44): 


$$
\bar{K}\left(\infty, E_{+}, E_{-}\right)=\frac{1}{2 v^{2}}\left[\sin ^{-1} \frac{E_{+} \sqrt{E_{+}^{2}-4 v^{2}}+E_{-} \sqrt{E_{-}^{2}-4 v^{2}}}{E_{+}^{2}+E_{-}^{2}-4 v^{2}}-\frac{\pi}{2}\right] .
$$

As before, the two particle Green function fails to factorize in energy. This has, however, a more serious consequence in the quantum Hall problem. Large momenta also correspond to large spatial separations in magnetic fields. More precisely, one has

$$
\left\langle G^{+}\left(\mathbf{r}, \mathbf{r}, E_{+}\right) G^{-}\left(\mathbf{0}, \mathbf{0}, E_{-}\right)\right\rangle=\int \frac{d^{2} q}{4 \pi^{2}} \exp \left(-\frac{1}{2 l^{2}}|\mathbf{z} \times \mathbf{r}+\mathbf{q} l|^{2}\right) \bar{K}\left(\mathbf{q}, E_{+}, E_{-}\right),
$$

and hence

$$
\lim _{\mathbf{r} \rightarrow \infty}\left\langle G\left(\mathbf{r}, \mathbf{r}, E_{+}\right) G\left(\mathbf{0}, \mathbf{0}, E_{-}\right)\right\rangle=\lim _{\mathbf{q} \rightarrow \infty} \frac{1}{2 \pi l^{2}} \bar{K}\left(\mathbf{q}, E_{+}, E_{-}\right) .
$$

So we discover that lack of energy factorization translates into a lack of factorization at infinite spatial separation, which is clearly unphysical. In addition, we again find that the retarded-retarded Green function is singular inside the impurity band in a limit where the exact answer is neccessarily analytic.

\section{SUMMARY AND OUTLOOK}

As this has been a largely technical discussion of the Liouvillian formalism, it is perhaps useful to summarize the main argument again. The work of SMG and MSZ has suggested that the critical divergence of the localization length for the (non-interacting) quantum Hall transition can be computed within the Liouvillian formalism by the $1 / N_{L}$ expansion in the number of Liouvillian flavors. We find, based on testing the Liouvillian approach on random martrix theory and energy unintegrating it at large momenta in the quantum Hall problem, that this program has serious problems already at $N_{L}=\infty$ which do not appear to be remediable within the $1 / N_{L}$ expansion. Accordingly, we conclude that the calculation of MSZ does not represent a computation of the quantum Hall correlation length exponent. As there isn't another approximation scheme that readily suggests itself in the Liouvillian formalism, we are pessimistic, as indicated by our title, about its utility for making progress on the problem of the quantum Hall transition.

That said, there are a couple of loose ends in our analysis that would be nice to tie up. First, we have not carried out the energy unintegration of the Liouvillian $N_{L}=\infty$ answer for the quantum Hall problem near $\mathbf{q}=0$ and it would be of some technical interest to see if that has an analytic structure different from large momenta as well as a technical challenge to see how that might get done. Second, it may be possible to use our ideas on energy unintegration to see if the logarithm found by MSZ has any interpretation in Green function perturbation theory. Perhaps an intrepid reader will be inspired to sort these out.

Finally, as we were finishing this work, there appeared a preprint 14 by Moore which studies the evolution of the QH Liouvillian as a function of $N_{L}$. Its most relevant finding, via numerical analysis at small $N_{L}$, is that the Liouvillian theory likely exhibits metallic diffusion, instead of critical scaling, at all $N_{L}>1$. Evidently, this is consistent with our own conclusion in Section IV D 3 that the theory for all $N_{L} \neq 1$ is unrepresentative of the original problem of interest $\left(N_{L}=1\right)$.

Acknowledgements: We have benefitted from numerous invigorating conversations with E. Fradkin, S. Girvin, S. Sachdev, I. Ussishkin and, especially, J. Moore. This research was supported in part by the National Science Foundation through grant No. DMR 99-78074 (VO and SLS), by the David and Lucille Packard Foundation (VO and SLS), and by the EPSRC under grant GR/J78327 (JTC).

\section{APPENDIX A: CRITICAL BEHAVIOR OF THE QUANTUM HALL LIOUVILLIAN}

For completeness, but also because some of the original discussion was incorrect 20 wiscuss here how it is that the energy integrated Liouvillian propagator can be used to extract the localization length exponent. One might worry that, as the critical wavefunctions are a set of mea- 
sure zero in the spectrum, any information on them will be lost upon integrating over the band and the behavior at small $\omega$ and $q$ will be that of an insulator. This is not the case: the Liouvillian Green function exhibits critical asymptotics intermediate between those of the metal and insulator in the transport limit $q \ll \omega$, with the diffusion constant $D(\omega, \mathbf{q}=\mathbf{0}) \sim \omega^{1 / 2 \nu}$, where $\nu$ is the correlation length exponent 20.

Near the critical energy $E_{c}$, the two particle Green function for the quantum Hall problem can be parametrized as 12

$$
K(E, \omega ; \mathbf{q})=\frac{2 \pi \rho(E)}{i \omega-D(E, \omega ; \mathbf{q}) q^{2}}
$$

In the transport limit $D q^{2} \ll \omega$ we can expand the denominator to obtain

$$
K(E, \omega ; \mathbf{q}) \approx 2 \pi \rho(E)\left[\frac{1}{i \omega}-\frac{D q^{2}}{\omega^{2}}\right] .
$$

These forms also hold for a metal, with $D(E, \omega ; \mathbf{q}=\mathbf{0})$ non-zero, and for an insulator with $D(E, \omega ; \mathbf{q}=\mathbf{0})$ vanishing for $\omega \rightarrow 0$ roughly as $\omega^{2}$. For the quantum Hall problem, $D(E, \omega, \mathbf{q})$ has a non-trivial dependence on $E$, $\omega$ and $\mathbf{q}$.
To quantify this dependence, consider the two important length scales in the transport limit. These are the localization length $\xi \sim l\left|E_{c} /\left(E-E_{c}\right)\right|^{\nu}$ and $L_{\omega}=$ $(\rho(\epsilon) \omega)^{-1 / 2}$ which is the size of a box with level spacing of order the frequency. Then, when $L_{\omega} \ll \xi(E)$, localization is unimportant and we may take $D(E, \omega, \mathbf{q}) \approx D_{c}$, a non-zero constant. In the opposite limit, $L_{\omega} \gg \xi(E)$, we have the insulating behavior, $D(E, \omega, \mathbf{q})=D_{\text {ins }}(\omega) \sim \omega^{2}$. In the following we will assume a sharp crossover between these two limiting forms, which is sufficient for our purposes.

Armed with these facts we can now carry out the energy integration to deduce the form of $\Pi(\mathbf{q}, \omega)$ in the transport limit. Specifically,

$$
\int d E \operatorname{Re} K(E, \omega ; \mathbf{q})=2 \pi \frac{q^{2}}{\omega^{2}} \int_{-\infty}^{\infty} d E \rho(E) D(E, \omega, \mathbf{q}=\mathbf{0}) .
$$

We will see that the integral is dominated by energies near $E_{c}$ so that we can replace $\rho(E) \approx \rho\left(E_{c}\right)$ and trade the integral over $E$ for one over the localization length using the asymptotic relation $\xi \sim l\left|E_{c} /\left(E-E_{c}\right)\right|^{\nu}$. This yields

$$
\begin{aligned}
\operatorname{Im} \Pi(\mathbf{q}, \omega) & \sim \frac{q^{2}}{\omega^{2}} \frac{E_{c} l^{1 / \nu}}{\nu} \rho\left(E_{c}\right) \int_{l}^{\infty} d \xi \xi^{-1-1 / \nu} D\left(E_{c}+E_{c}(l / \xi)^{1 / \nu}, \omega, \mathbf{q}=\mathbf{0}\right) \\
& \sim \frac{q^{2}}{\omega^{2}} \frac{E_{c} l^{1 / \nu}}{\nu} \rho\left(E_{c}\right)\left[\int_{l}^{L_{\omega}} d \xi \xi^{-1-1 / \nu} D_{\mathrm{ins}}(\omega)+\int_{L_{\omega}}^{\infty} d \xi \xi^{-1-1 / \nu} D_{c}\right] \\
& \sim \frac{q^{2}}{\omega^{2}} E_{c} \rho\left(E_{c}\right)\left[D_{\mathrm{ins}}(\omega)+D_{c}\left(l^{2} \rho\left(E_{c}\right) \omega\right)^{1 / 2 \nu}\right] \sim \frac{D_{c} q^{2}}{\omega^{2}}\left(l^{2} \rho\left(E_{c}\right) \omega\right)^{1 / 2 \nu}
\end{aligned}
$$

where the last simplifcation follows upon noting that $D_{\text {ins }}(\omega) \sim \omega^{2}$ is much smaller than $\omega^{\frac{1}{2 \nu}}$ for $\nu>1 / 4$, which is true in general of a random critical point in two dimensions on the grounds of the Harris criterion, and holds for the reasonably precise estimates of $\nu$ available in this case. As a statement about Liouvillian theory, this result implies that the self-energy of the exact Liouviliian Green function has the behavior $\Sigma(\omega, q \rightarrow 0)=$ $\omega-1 / \Pi(\omega, q) \sim i q^{2} \omega^{\frac{1}{2 \nu}}$.

\section{APPENDIX B: DETAILS OF ENERGY UNINTEGRATION}

We start from Eq. (43). After a change of variables, it reads

$$
\begin{aligned}
K\left(E_{+}, E_{-}\right) & =\int_{0}^{\frac{\pi}{2}} d \theta \int_{0}^{\infty} \frac{r d r \exp \left(-r\left(E_{-} \cos \theta+E_{+} \sin \theta\right)\right)}{2 v^{2} r^{2}} \oint \frac{d z e^{z}}{2 \pi i}\left[z-\sqrt{z^{2}-(2 v r)^{2}}\right] \\
& =\int_{0}^{\frac{\pi}{2}} d \theta \int_{0}^{\infty} \frac{d r \exp \left(-r\left(E_{-} \cos \theta+E_{+} \sin \theta\right)\right)}{v} I_{1}(2 v r) \\
& =\frac{1}{2 v^{2}} \int_{0}^{\frac{\pi}{2}} d \theta\left(\frac{R \cos (\theta+\phi)}{\sqrt{R^{2} \cos ^{2}(\theta+\phi)-1}}-1\right),
\end{aligned}
$$


where we have defined

$$
R \equiv \frac{1}{2 v} \sqrt{E_{+}^{2}+E_{-}^{2}}=\frac{1}{2 v} \sqrt{E^{2}+\left(\omega / 2+i 0^{+}\right)^{2}}
$$

and

$$
\tan \phi \equiv \frac{E_{-}}{E_{+}}
$$

Proceeding further,

$$
\begin{aligned}
K\left(E_{+}, E_{-}\right) & =\frac{1}{2 v^{2}}\left[\sin ^{-1} \frac{R \cos \phi}{\sqrt{R^{2}-1}}+\sin ^{-1} \frac{R \sin \phi}{\sqrt{R^{2}-1}}-\frac{\pi}{2}\right] \\
& =\frac{1}{2 v^{2}}\left[\sin ^{-1} \frac{E_{+}}{\sqrt{E_{+}^{2}+E_{-}^{2}-4 v^{2}}}+\sin ^{-1} \frac{E_{-}}{\sqrt{E_{+}^{2}+E_{-}^{2}-4 v^{2}}}-\frac{\pi}{2}\right] \\
& =\frac{1}{2 v^{2}}\left[\sin ^{-1} \frac{E_{+} \sqrt{E_{+}^{2}-4 v^{2}}+E_{-} \sqrt{E_{-}^{2}-4 v^{2}}}{E_{+}^{2}+E_{-}^{2}-4 v^{2}}-\frac{\pi}{2}\right]
\end{aligned}
$$

which is Eq. (44).

\section{APPENDIX C: MAGNETIC ALGEBRA AND RANDOM MATRICES}

In this appendix we demonstrate that an algebra like that of density operators in the lowest Landau level may be set up for an arbitrary Hamiltonian. We consider an orthonormal basis $\{|a\rangle\}$ for $\mathcal{V}$ and the corresponding basis $\{|a, b\rangle\}$ on $\mathcal{A}_{\mathcal{V}}$, where $a$ and $b$ range from 1 to $\left.N\right)$. We define

$$
\left.\mid \mathbf{q})=\frac{1}{N^{1 / 2}} e^{i q_{x} q_{y} \pi / N} \sum_{n} \mid n+q_{y}, n\right) e^{i q_{x} n 2 \pi / N}
$$

for integer $q_{x}$ and $q_{y}$ (ranging from $1 \rightarrow N$ ) which also form an orthonormal basis. We can rewrite

$$
\left.\left.\hat{H}=\sum_{m n} H_{m n} \mid m, n\right)=\sum_{\mathbf{q}} V_{-\mathbf{q}} \mid \mathbf{q}\right),
$$

where

$$
V_{-\mathbf{q}} \equiv \sum_{n} \frac{H_{n+q_{y}, n}}{N^{1 / 2}} e^{-i q_{x}\left(n+q_{y} / 2\right) 2 \pi / N}
$$

Switching to operator notation, with $\left.\tau_{\mathbf{q}} \equiv \mid \mathbf{q}\right)$, one can check that

$$
\left[\tau_{\mathbf{q}}, \tau_{\mathbf{p}}\right]=2 i \sin \frac{\pi \mathbf{q} \wedge \mathbf{p}}{N} \tau_{\mathbf{q}+\mathbf{p}}
$$

so that

$$
\left.\left.\frac{\partial}{i \partial t} \mid \mathbf{q}\right)=\frac{1}{N^{1 / 2}} \sum_{\mathbf{p}} 2 i V_{-\mathbf{p}} \sin \frac{\pi \mathbf{q} \wedge \mathbf{p}}{N} \mid \mathbf{p}+\mathbf{q}\right)
$$

These expressions parallel those for the Liouvillian in the lowest Landau level. Strikingly we have recast a generic problem in the notation of the magnetic translation operators. Of course, the definition of the (discrete) momenta is adhoc, and so, while this rewriting appears suggestive, it is not generally useful. Nevertheless, the GUE retains its simplicity in this approach: Eq. 27) implies that

$$
\left\langle V_{\mathbf{p}} V_{\mathbf{q}}\right\rangle=\frac{v^{2}}{N} \delta_{\mathbf{p},-\mathbf{q}}
$$

Disorder-correlators are thus diagonal in $\mathbf{q}$, continuing the analogy with spatially extended systems.
1 For a review, see: B. Huckestein, Rev. Mod. Phys. 67, 357-396 (1995).

2 H. Levine, S. B. Libby, and A. M. M. Pruisken, Phys. Rev.
Lett. 51, 1915-1918 (1983).

3 D. E. Khmelnitskii, JETP Lett. 38, 552 (1983).

${ }^{4}$ H. P. Wei, D. C. Tsui and A. M. M. Pruisken, Phys Rev B 
331488 (1986); H. P. Wei, D. C. Tsui, M. Paalanen and A. M. M. Pruisken, Phys Rev Lett 611294 (1988); L. W. Engel, D. Shahar, C. Kurdak, and D. C. Tsui, Phys. Rev. Lett. 71, 2638 (1993).

${ }^{5}$ F. Hohls, U. Zeitler, and R. J. Haug, Phys. Rev. Lett. 86, 5124-5127 (2001); Phys. Rev. Lett. 88, 036802 (2002); Phys. Rev. B 66, 073304 (2002).

${ }^{6}$ D. Shahar, M. Hilke, C. C. Li, D. C. Tsui, S. L. Sondhi, J. E. Cunningham, and M. Razeghi, Solid State Comm. 107, 19 (1998).

7 J. Sinova, V. Meden, and S. M. Girvin, Phys. Rev. B 62, 2008 (2000).

8 J. E. Moore, J. Sinova and A. Zee, Phys. Rev. Lett. 87, 046801 (2001).

${ }^{9}$ For other work on the Liovillian approach, not directly relevant to the line of argument in this paper, see V. Gurarie and A. Zee, Int. J. B 15, 1225 (2001).

10 R. Oppermann and F. Wegner, Z. Phys. B 34, 327 (1979).

11 Oleg Tchernyshyov, private communication.

12 J. T. Chalker, Physica A 167, 253 (1990).

13 The N-orbital generalisation of the quantum Hall localization problem, introduced in T. S. J. Streit, J. Phys. Lett. (Paris) 45, L713-L718 (1984) is expected to have N plateau transitions. See D. K. K. Lee and J. T. Chalker Phys. Rev. Lett. 72, 1510-1513 (1994) and Z. Wang, D. Lee, and X.
Wen Phys. Rev. Lett. 72, 2454-2457 (1994).

14 J. E. Moore, "Conservation laws in the quantum Hall Liouvillian theory and its generalizations", cond-mat/0209299.

15 F. Wegner, Z. Phys. B 51, 279 (1983).

16 S. Hikami, Phys. Rev. B 29, 3726 (1984); R. R. P. Singh,S. Chakravarty, Nucl. Phys. B265, 265 (1986).

17 One easy way to see that this is true is by assuming no dependence at large momenta and verify self-consistency of this assumption. More systematicly, the corrections are Gaussianly small.

18 See for instance J. T. Chalker, J. Phys. C 20, L493, (1987).

19 LDOS can be computed exactly for $N=2$ and $N_{L}=1$ :

$$
\begin{array}{ll}
\text { GOE : } & \Omega(\omega)=2 \delta(\omega)+\frac{|\omega|}{v^{2}} e^{-\frac{\omega^{2}}{2 v^{2}}} \\
\text { GUE : } & \Omega(\omega)=2 \delta(\omega)+\frac{4 \omega^{2}}{\sqrt{\pi} v^{3}} e^{-\frac{\omega^{2}}{v^{2}}}
\end{array}
$$

Note that Eq. (27) is equivalent, for general $N$, to matrices being distributed according to $\mathcal{P}[\hat{H}] \sim$ $\exp \left[-\frac{N}{(1+\alpha) v^{2}} \operatorname{Tr}\left\{\hat{H}^{2}\right\}\right]$.

20 SMG proposed instead a wavevector dependent $D(q) \sim$ $q^{\frac{1}{\nu}}$. This was corrected later by Gurarie and Zee 9 . 